\title{
Deep Exploration of the Planets HR 8799 b, c, and d with Moderate-resolution Spectroscopy
}

\author{
Jean-Baptiste Ruffio $^{1,2}$ (D), Quinn M. Konopacky ${ }^{3}$ (D), Travis Barman ${ }^{4}$ (D), Bruce Macintosh ${ }^{2}$ (D), Kielan K. W. Hoch ${ }^{3}$ (D), \\ Robert J. De Rosa ${ }^{5}$ (D), Jason J. Wang ${ }^{1,13}$ (iD), Ian Czekala ${ }^{6,7,8,9,10,14}$ (i), and Christian Marois ${ }^{11,12}$ (ID) \\ ${ }^{1}$ Department of Astronomy, California Institute of Technology, Pasadena, CA 91125, USA; jruffio@ caltech.edu \\ ${ }^{2}$ Kavli Institute for Particle Astrophysics and Cosmology, Stanford University, Stanford, CA 94305, USA \\ ${ }^{3}$ Center for Astrophysics and Space Science, University of California, San Diego; La Jolla, CA 92093, USA \\ ${ }^{4}$ Lunar and Planetary Laboratory, University of Arizona, Tucson, AZ 85721, USA \\ ${ }^{5}$ European Southern Observatory, Alonso de Córdova 3107, Vitacura, Santiago, Chile \\ ${ }_{7}^{6}$ Department of Astronomy and Astrophysics, 525 Davey Laboratory, The Pennsylvania State University, University Park, PA 16802, USA \\ ${ }^{7}$ Center for Exoplanets and Habitable Worlds, 525 Davey Laboratory, The Pennsylvania State University, University Park, PA 16802, USA \\ ${ }^{8}$ Center for Astrostatistics, 525 Davey Laboratory, The Pennsylvania State University, University Park, PA 16802, USA \\ ${ }^{9}$ Institute for Computational \& Data Sciences, The Pennsylvania State University, University Park, PA 16802, USA \\ ${ }_{10}^{10}$ Department of Astronomy, 501 Campbell Hall, University of California, Berkeley, CA 94720-3411, USA \\ ${ }^{11}$ National Research Council of Canada Herzberg, 5071 West Saanich Rd, Victoria, BC, V9E 2E7, Canada \\ ${ }^{12}$ University of Victoria, 3800 Finnerty Rd, Victoria, BC, V8P 5C2, Canada \\ Received 2021 February 9; revised 2021 September 5; accepted 2021 September 14; published 2021 December 6
}

\begin{abstract}
The four directly imaged planets orbiting the star HR 8799 are an ideal laboratory to probe atmospheric physics and formation models. We present more than a decade's worth of Keck/OSIRIS observations of these planets, which represent the most detailed look at their atmospheres to date by its resolution and signal-to-noise ratio. We present the first direct detection of HR $8799 \mathrm{~d}$, the second-closest known planet to the star, at moderate spectral resolution with Keck/OSIRIS ( $K$ band; $R \approx 4000$ ). Additionally, we uniformly analyze new and archival OSIRIS data ( $H$ and $K$ band) of HR 8799 b, c, and d. First, we show detections of water $\left(\mathrm{H}_{2} \mathrm{O}\right)$ and carbon monoxide (CO) in the three planets and discuss the ambiguous case of methane $\left(\mathrm{CH}_{4}\right)$ in the atmosphere of $\mathrm{HR} 8799 \mathrm{~b}$. Then, we report radial-velocity (RV) measurements for each of the three planets. The RV measurement of HR $8799 \mathrm{~d}$ is consistent with predictions made assuming coplanarity and orbital stability of the HR 8799 planetary system. Finally, we perform a uniform atmospheric analysis on the OSIRIS data, published photometric points, and lowresolution spectra. We do not infer any significant deviation from the stellar value of the carbon-to-oxygen ratio $(\mathrm{C} / \mathrm{O})$ of the three planets, which therefore does not yet yield definitive information about the location or method of formation. However, constraining the C/O for all the HR 8799 planets is a milestone for any multiplanet system, and particularly important for large, widely separated gas giants with uncertain formation processes.
\end{abstract}

Unified Astronomy Thesaurus concepts: Extrasolar gaseous giant planets (509); Exoplanet atmospheric composition (2021); Direct imaging (387); Exoplanet formation (492)

Supporting material: data behind figure

\section{Introduction}

There are two main pathways to form substellar companions around young stars (Lissauer 1987; Boss 1997; Boley 2009). On one hand, planets like those in our solar system are believed to form by core accretion, in which a core slowly accretes solid material before reaching a critical mass that triggers runaway accretion of the gas. On the other hand, substellar companions can, for example, form by gravitational instability of the protoplanetary disk or turbulent fragmentation of the protostellar cloud. In the transitional mass range between planets and brown dwarfs, the formation mechanisms of directly imaged planets ( $>2 M_{\text {Jup }}$; effective temperature $500-2000 \mathrm{~K}$; semimajor axis $>5 \mathrm{au}$ ) are still outstanding (Kratter et al. 2010; Nielsen et al. 2019). The present-day composition of a planet's atmosphere may contain evidence that can be used to probe its formation history, including its original location, migration pathways, and the fraction of gas and solids it accreted (Öberg et al. 2011). Indeed, following the core accretion paradigm, the composition of planetesimals in the protoplanetary disk is

\footnotetext{
${ }^{13} 51$ Pegasi b Fellow.

${ }^{14}$ NASA Hubble Fellowship Program Sagan Fellow.
}

controlled by the different ice lines, where volatiles freeze out of the gas phase. Alternatively, objects forming through gravitational instability are expected to inherit the bulk composition of the original protostellar gas, giving the same composition as the star. For these reasons, abundance ratios such as the carbon-tooxygen ratio $(\mathrm{C} / \mathrm{O})$ are considered to be important tracers of planet formation. Evidently, other factors, like the efficiency of the mixing within the planet's atmosphere, planetesimal drift, migration, and core dredging, could also strongly influence the final composition of an atmosphere (Mordasini et al. 2016; Madhusudhan et al. 2017).

The ability of high-contrast imaging instruments to spatially resolve the light of the planet from the star makes direct imaging an excellent way to characterize exoplanets. The system of four planets (10-70 au, 5-13 $M_{\text {Jup }}$ ) orbiting HR 8799 was the first multiplanet system to be directly imaged (Marois et al. 2008, 2010). The host star is $\sim 1.5 M_{\odot}$ (Gray \& Kaye 1999; Baines et al. 2012), 42 Myr old (Zuckerman et al. 2011; Malo et al. 2013; Bell et al. 2015), and is located at $41.3 \pm 0.2 \mathrm{pc}$ (Gaia Collaboration 2018). From orbital monitoring and modeling, the four planets are thought to be within a few degrees of coplanarity, and at or near 1:2:4:8 resonance 
(Fabrycky \& Murray-Clay 2010; Soummer et al. 2011; Pueyo et al. 2015; Konopacky et al. 2016; Zurlo et al. 2016; Wertz et al. 2017; Wang et al. 2018b; Gravity Collaboration et al. 2019; O'Neil et al. 2019; Ruffio et al. 2019; Goździewski \& Migaszewski 2020). They are bracketed by both a warm and a cold debris disk similar to the asteroid and Kuiper Belts in the Solar system (Reidemeister et al. 2009; Su et al. 2009; Hughes et al. 2011; Matthews et al. 2014; Booth et al. 2016; Contro et al. 2016; Read et al. 2018; Goździewski \& Migaszewski 2018; Wilner et al. 2018; Geiler et al. 2019). HR 8799 b, c, d, and e, which have been observed with a broad wavelength coverage (1-5 $\mu \mathrm{m}$ ) and spectral resolution up to $R=15,000$ (Wang et al. 2018a), are among the best studied exoplanets and as such represent a unique opportunity for comparative planetology. From low-resolution spectroscopy and photometry, the planets were found to significantly differ from field brown dwarfs of similar effective temperature (Bowler et al. 2010; Currie et al. 2011; Madhusudhan et al. 2011; Skemer et al. 2014; Bonnefoy et al. 2016; Zurlo et al. 2016). They likely feature thick, patchy clouds, and disequilibrium chemistry (Hinz et al. 2010; Galicher et al. 2011; Skemer et al. 2012; Currie et al. 2014; Ingraham et al. 2014; Greenbaum et al. 2018).

At low spectral resolution $(R=\lambda / \Delta \lambda \approx 30)$, quantitative assessment of planetary molecular abundances is limited by degeneracies between abundances, clouds, and pressure or gravity. Higher resolution spectra can help break these degeneracies. Moderate $(R \sim 4000)$ and high $(R>25,000)$ resolution spectroscopy can resolve individual absorption lines, providing unambiguous detection of specific molecules and their abundance. Additionally, spectroscopy of substellar companions at these resolutions has enabled radial-velocity (RV) measurements (Brogi et al. 2014; Snellen et al. 2014; Ruffio et al. 2019), spin measurements (Lissauer \& Kary 1991; Snellen et al. 2014; Schwarz et al. 2016; Bryan et al. 2018, 2020), and the creation of "surface" maps (through Doppler mapping; Crossfield 2014; Crossfield et al. 2014).

Infrared moderate-resolution spectroscopy with Keck/OSIRIS $(R \approx 4000$; Larkin et al. 2006) has allowed the unambiguous detection of water $\left(\mathrm{H}_{2} \mathrm{O}\right)$ and carbon monoxide (CO; Konopacky et al. 2013; Barman et al. 2015). While methane $\left(\mathrm{CH}_{4}\right)$ is abundant in the atmosphere of cool field brown dwarfs, its lack of strong detection in the atmosphere of the HR 8799 planets is evidence of disequilibrium chemistry Barman et al. (2011a). An initial detection of $\mathrm{CH}_{4}$ in HR 8799 b by Barman et al. (2015) was not confirmed by Petit dit de la Roche et al. (2018). Further analyses and observations are therefore warranted to confirm the abundance of methane and the magnitude of the eddy diffusion coefficient. Moderate-resolution spectroscopy in $K$ band is particularly useful estimate to the $\mathrm{C} / \mathrm{O}$ of an exoplanet, which can then be compared to the $\mathrm{C} / \mathrm{O}$ of the star. The $\mathrm{C} / \mathrm{O}$ of the host star HR 8799 is close to solar with $\mathrm{C} / \mathrm{O}=0.54_{-0.09}^{+0.12}$ (Wang et al. 2020). Using OSIRIS spectra and self-consistent atmospheric models, slightly super stellar $\mathrm{C} / \mathrm{O}$ were found for $\mathrm{HR}$ $8799 \mathrm{~b}$ and c, $[\mathrm{C} / \mathrm{O}]_{\mathrm{c}}=0.65_{-0.05}^{+0.1}$ Konopacky et al. (2013) and $[\mathrm{C} / \mathrm{O}]_{\mathrm{b}} \in[0.58,0.7]$ Barman et al. (2015), suggesting core accretion scenarios. However, there exists a significant scatter in $\mathrm{C} / \mathrm{O}$ estimates between different studies. There exists two complementary paradigms for atmospheric characterization: precomputed self-consistent forward models, and atmospheric retrievals which fit for the temperature-pressure profile but do not ensure overall chemical equilibrium. Due to the computational cost of generating models at high spectral resolution, forward model grids are useful for high-resolution data, but remain limited by the small number of varied parameters and a coarse sampling. Atmospheric retrievals of lower resolution spectra infer a C/O near unity for HR $8799 \mathrm{~b}$, but stellar C/O for the other three planets: $[\mathrm{C} / \mathrm{O}]_{\mathrm{b}}=0.97 \pm 0.01$ (Lee et al. 2013), $[\mathrm{C} / \mathrm{O}]_{\mathrm{b}}=0.92 \pm 0.01$ and $[\mathrm{C} / \mathrm{O}]_{\mathrm{c}}=0.55 \pm 0.1$ (Lavie et al. 2017), $[\mathrm{C} / \mathrm{O}]_{e}=0.6_{-0.08}^{+0.07}$ (Mollière et al. 2020), and $[\mathrm{C} / \mathrm{O}]_{\mathrm{c}}=0.58_{-0.06}^{0.06}$ with weak priors (Wang et al. 2020). Wang et al. (2020) also shows that the C/O can vary significantly, from 0.39 to 0.58 in this case, depending on the priors used and the data included in the fit of HR $8799 \mathrm{c}$.

Methods to measure stellar radial velocities for exoplanet Doppler detection are generally classified into two categories: cross correlation (e.g., Baranne et al. 1996) and forward modeling (e.g., Gao et al. 2016; Cale et al. 2019); although both techniques fundamentally rely on likelihood maximization. Exoplanet atmospheres have mostly been studied at high spectral resolution spectroscopy using the simpler cross correlation function technique. In Ruffio et al. (2019), we developed a forward model for high-contrast companions at high spectral resolution. Forward modeling relies on minimal preprocessing of the data, and allows the direct estimation of any atmospheric parameters (RV, C/O, etc.) with their associated uncertainties. The joint estimation of the planet signal and the starlight avoids the oversubtraction of the planet signal when subtracting the starlight. Another significant advantage is the uncertainties corresponding to the starlight subtraction can be analytically marginalized out of the joint posterior probability distribution. We note that in the presence of flat priors, this method and the conventional cross correlation function are both maximum-likelihood estimations of the planet signal. Using the forward modeling approach, we were able to measure the RV of HR $8799 \mathrm{~b}$ and c, and resolve the 3D orientation of the system (Ruffio et al. 2019). This new framework allowed a first preliminary detection of HR $8799 \mathrm{~d}$ at moderate spectral resolution in Ruffio (2019). The framework was improved in Wilcomb et al. (2020) to correct for systematics and account for intranight telluric variability.

In this work, we present new deep HR 8799d observations and perform a uniform analysis of the three planets. First, we describe the observations and data reduction in Section 2, and redefine the statistical model in Section 3. The detection of $\mathrm{H}_{2} \mathrm{O}, \mathrm{CO}$, and $\mathrm{CH}_{4}$ is discussed in Section 4 along with the extracted spectra for the three planets. Then, we present the first RV measurement of HR 8799d and revise the RV of HR 8799 $\mathrm{b}$ and $\mathrm{c}$ in Section 5. In Section 7, we fit a grid of atmospheric models, including a parameterization of $\mathrm{C} / \mathrm{O}$, to the OSIRIS data as well as photometric data points and lower resolution spectra. Finally, we discuss the results in Section 8 and conclude in Section 9.

\section{Observations}

OSIRIS is an infrared integral field spectrograph on the W. M. Keck I telescope with moderate spectral resolution $(R=\lambda$ / $d \lambda \sim 4000$; Larkin et al. 2006; Mieda et al. 2014; Boehle et al. 2016; Lockhart et al. 2019). The smallest platescale (20 mas pix $^{-1} ; \sim 1$ !" $3 \times 0$ "! 3 field of view) was used for all but the 2018 observations $\left(35\right.$ mas pix $\left.^{-1}\right)$. The spectral cubes were reduced using the OSIRIS data reduction pipeline (Krabbe et al. 2004; Lyke et al. 2017).

Observing logs for the science and the telluric standards can be found in Appendix A. The wavelength, transmission, and 
point-spread function (PSF) calibration are described in detail in Ruffio et al. (2019). The wavelength solution was calibrated using the $\mathrm{OH}^{-}$emission lines from the sky observations. Standard star observations are used to calibrate a supersampled PSF and the transmission spectrum, which are subsequently used in the forward model of the data. The adaptive optics correction was sometimes turned off to avoid detector persistence. In this case, or when the PSF was truncated, the spectrum was only used to calibrate the transmission. In 2020, we changed to observing strategy and started only using the host star for calibration. Persistence issues were avoided by only integrating the star at the edge of the field of view and avoiding the center where the planet would be located.

Using the method described below, the planets were detected in a number of exposures totaling $12.7 \mathrm{hr}$ out of $14.4 \mathrm{hr}$ integrated on HR $8799 \mathrm{~b}$ in $K$ band $(1.97-2.38 \mu \mathrm{m})$ and 0.8 out of $6.8 \mathrm{hr}$ in $H$ band $(1.47-1.80 \mu \mathrm{m}), 7.7$ out of $8.3 \mathrm{hr}$ in $K$ band and 2.8 out of $9.5 \mathrm{hr}$ in $H$ band for HR $8799 \mathrm{c}$, and finally 9.2 out of $20.0 \mathrm{hr}$ in $K$ band for HR $8799 \mathrm{~d}$. We can only use exposures in which the planet is detected for any subsequent analysis because OSIRIS pointing and dithering precision does not allow a blind localization of the planet. While a subset of this data has already been published (Barman et al. 2011a; Konopacky et al. 2013), the $H$-band data on HR $8799 \mathrm{c}$ and the $K$-band data for HR $8799 \mathrm{~d}$ are presented here for the first time.

\section{Data Modeling}

We use the method developed in Ruffio et al. (2019) and improved in Wilcomb et al. (2020). The general concept is to define a linear model of the data that includes the planet signal, the starlight, and a set of principal components of the residuals to include possible systematics. The linear model can be easily optimized and analytically marginalized to quickly calculate the posterior probability density of any other nonlinear parameters, such as RV, temperature, surface gravity, and C/ O. For completeness, we redefine the model below, such that

$$
\boldsymbol{d}=\boldsymbol{M \phi}+\boldsymbol{n}
$$

where $\boldsymbol{d}$ is the data, $\boldsymbol{M}$ is the linear model (which is itself a function of nonlinear parameters, e.g., RV, C/O, etc.), $\phi$ are the linear parameters, and $\boldsymbol{n}$ is the noise. Each term is described in the following subsections in more detail.

\subsection{The Data}

The data vector $\boldsymbol{d}$ contains the pixels of a postage-stampsized spectral cube, centered at the location of interest with $5 \times 5$ spaxels in the spatial dimensions and $N_{\lambda}=1665$ pixels in the wavelength direction in $K$ band. The spectrum of each spaxel is high-pass filtered using a periodicity cutoff of 83 pix ( i.e., $21 \mathrm{~nm}$ ) in Fourier space (see Ruffio et al. 2019). Bad pixels are simply removed from the data vector; they are identified by the OSIRIS data reduction pipeline and additional sigma clipping.

\subsection{The Noise}

We assume uncorrelated centered Gaussian noise, which means that the random vector $\boldsymbol{n}$ has zero mean, and it is described by a diagonal covariance matrix $\Sigma=s^{2} \Sigma_{0} . \Sigma_{0}$ is a fixed diagonal matrix containing a quantity proportional to the error at each pixel, and $s$ is a scaling factor that is fitted for. In the following, inferred parameters are marginalized over the scaling factor. The diagonal elements of $\Sigma_{0}$ are defined as the square root of the continuum of the spectrum at each location. The values are clipped at half their median to avoid vanishing errors that would bias the result.

\subsection{The Model Matrix}

The model of the signal includes a representation of the companion, the starlight at the location of the companion, and a set of principal components used to account for the imperfection of the telluric model or other systematics. The latter is a way to account for the time variability of the tellurics when using limited standard star observations. It is defined by the $N_{\mathrm{d}} \times N_{\boldsymbol{\phi}}$ matrix $\boldsymbol{M}$, where $N_{d}$ is the size of $\boldsymbol{d}$ and $N_{\boldsymbol{\phi}}$ is the number of linear parameters,

$$
\boldsymbol{M}=\left[\boldsymbol{c}_{0, \mathrm{planet}}, \boldsymbol{c}_{1}, \ldots, \boldsymbol{c}_{25}, \boldsymbol{r}_{\mathrm{pc} 1}, \ldots, \boldsymbol{r}_{\mathrm{pc} K}\right]
$$

The first column vector $\boldsymbol{c}_{0 \text {,planet }}$ represents the planet signal. It is defined as the instrument PSF scaled by an atmospheric model of the companion and multiplied by the transmission of the atmosphere and the instrument. The next 25 column vectors, $\left\{c_{i}\right\}$ with $1 \leqslant i \leqslant 25$, represent the starlight spectrum at the $5 \times 5$ spaxels centered at the location of the companion. The starlight spectra need to be scaled to match the intensity and chromaticity of the speckle field at each location. With $i$ being the spaxel index, the starlight is defined as

$$
\boldsymbol{c}_{i}=\boldsymbol{d}_{i, L} \frac{\left(\mathcal{T S}_{\mathrm{star}}\right)_{H}}{\left(\mathcal{T S}_{\mathrm{star}}\right)_{L}}
$$

where $\boldsymbol{d}_{i, L}$ is the low-pass-filtered spectrum of the data for the spaxel $i, \mathcal{T}$ is the transmission of the atmosphere and the instrument, and $\mathcal{S}_{\text {star }}$ is the model spectrum of the star from the Phoenix library (Husser et al. 2013). The subscript $H$ and $L$ respectively designate the high-pass- and low-pass-filtered version of the spectrum.

The last $K$-column vectors $\left\{\boldsymbol{r}_{\mathrm{pc} i}\right\}$ are defined from the principal components of the residuals of a preliminary fit following the recipe below (Wilcomb et al. 2020). The preprocessing steps include:

1. At each location in the field of view, we fit and subtract the model with the starlight components only.

2. Then we extract a spectrum from the $5 \times 5 \times N_{\lambda}$ data cube of residuals by fitting the PSF at each wavelength.

3. The spectrum is normalized to the local continuum by dividing it by the low-pass-filtered data at the same location.

4. The principal components are then calculated from the resulting normalized spectra over the entire field of view, but excluding the region within five spaxels of the real companion.

For any subsequent modeling of the data and at any location of interest:

1. The principal components are scaled back to the local continuum.

2. Then, the $1 \mathrm{D}$ principal component spectra are applied to the 3D PSF model resulting in $K$-column vectors $\left(\boldsymbol{r}_{\mathrm{pc} 1}, \ldots, \boldsymbol{r}_{\mathrm{pcK}}\right)$ matching the dimensions of the data $\boldsymbol{d}$. 
This shows the flexibility of the linear model that we defined previously. Indeed, the model can be adapted or improved at will without changing the general framework. One advantage of this approach, compared to directly subtracting the projection of the data on a set of principal components (Hoeijmakers et al. 2018; Petit dit de la Roche et al. 2018; Wang et al. 2018a), is to avoid the oversubtraction of the planet signal. For example, oversubtraction at moderate resolution could occur when telluric lines that appear in the principal components could subtract real molecular features from the planet, hence biasing subsequent abundance estimates. In the following, we use $K=10$ principal components as in Wilcomb et al. (2020). The forward model remains sensitive to the shape of the continuum of the spectrum despite the fact that the spectra are high-pass filtered. Indeed, the shape of the continuum remains encoded in the depth of the different spectral lines in the atmospheric model and the tellurics.

\subsection{The Parameters}

The parameters of interest are the radial velocity of the companion and the atmospheric parameters: temperature $(T)$, surface gravity $(\log (g))$, and the ratio of carbon abundance to oxygen abundance $(\mathrm{C} / \mathrm{O})$. These are nonlinear parameters, grouped in the symbol $\psi$, that define the companion component $\boldsymbol{c}_{0 \text {,planet }}$ in the matrix $\boldsymbol{M}$. Their estimation and the calculation of the posteriors are discussed in Section 3.7. For simplicity, the atmospheric model is fixed when trying to detect the location of the planet and when estimating its radial velocity.

Then, there are $1+25+K$ linear parameters in $\phi$, which correspond to the amplitude of the companion signal, the amplitude of the starlight at each of the $5 \times 5$ spaxels centered at the position of the companion, and the amplitude of the $\mathrm{K}$ principal components. The last parameter is the scaling factor, $s$, of the noise. The linear parameters, as well as the noise scaling factor, are all nuisance parameters that will be marginalized over when estimating the nonlinear parameters.

\subsection{The Likelihood Function}

The likelihood function is defined as

$$
\begin{aligned}
& \mathcal{L}\left(\boldsymbol{\psi}, \boldsymbol{\phi}, s^{2}\right)=\frac{1}{\sqrt{(2 \pi)^{N_{\mathrm{d}}}\left|\boldsymbol{\Sigma}_{0}\right| s^{2 N_{\mathrm{d}}}}} \\
& \quad \times \exp \left\{-\frac{1}{2 s^{2}}\left(\boldsymbol{d}-\boldsymbol{M}_{\boldsymbol{\psi}} \boldsymbol{\phi}\right)^{\top} \boldsymbol{\Sigma}_{0}^{-1}\left(\boldsymbol{d}-\boldsymbol{M}_{\boldsymbol{\psi}} \boldsymbol{\phi}\right)\right\} .
\end{aligned}
$$

We also define $\chi^{2}=\chi_{0}^{2} / s^{2}$, with

$$
\chi_{0}^{2}=(\boldsymbol{d}-\boldsymbol{M} \phi)^{\top} \boldsymbol{\Sigma}_{0}^{-1}(\boldsymbol{d}-\boldsymbol{M} \boldsymbol{\phi}) .
$$

This likelihood will be used to:

1. detect the planet in the raw spectral cubes;

2. directly detect specific molecules in their atmospheres;

3. and estimate the $\mathrm{RV}$ and the $\mathrm{C} / \mathrm{O}$ of the three planets.

We briefly describe the methods in the following sections (see Appendix of Ruffio et al. 2019 for complete mathematical derivations).

\subsection{Planet and Molecule Detection}

OSIRIS is an integral field spectrograph, and the lack of precise pointing requires the detection of the companion in individual exposures before they can be combined. We choose an approach comparable to the cross-correlation function (CCF) for the detection of the planet itself as well as molecules in its atmosphere.

As discussed in Ruffio et al. (2019), the commonly used CCF is a simplified maximum-likelihood approach in which one estimates the signal-to-noise ratio $(\mathrm{S} / \mathrm{N})$ of the amplitude of the molecular spectral template in the data. We can produce a similar output using the data model defined previously by extracting the amplitude of the companion component, which is the first element of the $\phi$ vector, and its associated error. Using the model defined in this Section 3, the $\mathrm{S} / \mathrm{N}$ of the companion is defined as

$$
\mathrm{S} / \mathrm{N}=\frac{\tilde{\phi}[0]}{\sqrt{\operatorname{cov}(\tilde{\phi})[0,0]}} .
$$

The tilde signifies that the linear parameters $\phi$ is set to its optimal value with

$$
\tilde{\boldsymbol{\phi}}=\left(\boldsymbol{M}^{\top} \boldsymbol{\Sigma}_{0}^{-1} \boldsymbol{M}\right)^{-1} \boldsymbol{M}^{\top} \boldsymbol{\Sigma}_{0}^{-1} \boldsymbol{d},
$$

although it is numerically faster and more stable to estimate $\tilde{\phi}$ from a linear least square solver that does not require matrix inversion. The covariance matrix is defined as

$$
\operatorname{cov}(\tilde{\boldsymbol{\phi}})=\tilde{s}^{2}\left(\boldsymbol{M}^{\top} \boldsymbol{\Sigma}_{0}^{-1} \boldsymbol{M}\right)^{-1},
$$

and

$$
\tilde{s}^{2}=\frac{1}{N_{\mathrm{d}}} \chi_{0, \phi=\tilde{\phi}}^{2} .
$$

The $\mathrm{S} / \mathrm{N}$ from Equation (6) is computed at each spaxel location in the field of view and for a set of RV shifts of the companion spectrum resulting in a $\mathrm{S} / \mathrm{N}$ cube. The $\mathrm{S} / \mathrm{N}$ defined in Equation (6) might not always be accurate due to the simplifying assumptions in the modeling the noise. As a mitigation strategy, we subtract the median of the $\mathrm{S} / \mathrm{N}$ cube in the RV dimension and then in the spatial direction. In a similar fashion, we divide the $\mathrm{S} / \mathrm{N}$ by the sample standard deviation computed at each location using the spectral direction. Then, individual $\mathrm{S} / \mathrm{N}$ cubes for each exposures are registered and added together into a final cube for each planet. We finally ensure that the final $\mathrm{S} / \mathrm{N}$ cube as a whole has a unit standard deviation after masking the locations within five pixels $(\sim 0$ ". 1$)$ of the companion. The standard deviation calculations are performed after masking the spaxels at the locations of the planet.

For the detection of the planet, we use the best-fit atmospheric models described in Barman et al. (2015) and Konopacky et al. (2013) as spectral templates. The same template is used for HR 8799d as for HR 8799 c, which is justified by the similar atmospheric parameters retrieved in Section 7.2. The $\mathrm{S} / \mathrm{N}$ maps at the expected RV of the star for each HR 8799d exposure are shown in Appendix A for 10 principal components. In Appendix B, we show that the forward model is able to detect HR 8799d while it is $\sim 30$ times fainter than the starlight at the same location in only 10 minutes. The residuals are consistent with the photon noise limit and the planet $\mathrm{S} / \mathrm{N}$ per bin is $\sim 2$.

Once the planet's location is known, molecules are detected using the molecular templates described in Konopacky et al. (2013), Barman et al. (2015). The final $\mathrm{S} / \mathrm{N}$ curves as a 
function of the RV shift of the molecular spectra are presented in Section 4.

\subsection{Inference}

In this work, we are defining two inference problems: first, the estimation of the RV of the companion assuming a fixed atmospheric model; and second, the estimation of the atmospheric parameters while marginalizing over the RV. The parameters of the atmospheric models are: effective temperature $\left(T_{\text {eff }}\right)$, surface gravity $\left(\log (g)\right.$; with $g$ in $\left.\mathrm{cm} \mathrm{s}^{-2}\right)$, and carbon-to-oxygen ratio $(\mathrm{C} / \mathrm{O})$. In both cases, we also marginalize over the linear parameters $\phi$ and the noise scaling parameter $s$; this can be done analytically (Ruffio et al. 2019). Although it is technically possible, we do not marginalize over the location of the planet and assumed it fixed once the planet is detected. If the parameters of interest are represented by a vector $\psi$, where $\psi=[\mathrm{RV}]$ or $\psi=\left[T_{\mathrm{eff}}, \log (g), \mathrm{C} / \mathrm{O}, \mathrm{RV}\right]$, its posterior can be written as

$$
\mathcal{P}(\boldsymbol{\psi} \mid \boldsymbol{d}) \propto \frac{\mathcal{P}(\boldsymbol{\psi})}{\sqrt{\left|\boldsymbol{\Sigma}_{0}\right| \times\left|\boldsymbol{M}_{\psi}^{\top} \boldsymbol{\Sigma}_{0}^{-1} \boldsymbol{M}_{\boldsymbol{\psi}}\right|}}\left(\frac{1}{\chi_{0, \phi=\tilde{\phi}, \psi}^{2}}\right)^{\frac{N_{d}-N_{\phi}+1}{2}},
$$

where $\mathcal{P}(\psi)$ is $\psi$ 's prior. The model and the number of parameters are such that it is computationally tractable to calculate the posterior on a discrete grid of the parameter space. $\mathcal{P}(\psi)$ is assumed to be flat over the finite extent of the grid. Uniform improper priors are also assumed for the linear parameters $\phi$. We have assumed the inverse squared prior for the scaling parameter $s$ which corresponds to Jeffrey's prior when jointly estimating the mean and standard deviation of a Gaussian distribution. In the following, we express estimated parameters as the mode of the posterior distribution, and the error bars as the $68 \%$ confidence interval that includes the highest values of the probability distribution function.

\section{Detection of Molecules}

We use the maximum-likelihood-based method described in Section 3.6 to compute the $\mathrm{S} / \mathrm{N}$ of a spectral template as a function of its RV shift, which is conceptually comparable to a CCF. For each planet, we use four different templates: a full atmospheric model, which is both used for the detection of the planet and its RV estimation, and templates for $\mathrm{H}_{2} \mathrm{O}, \mathrm{CO}$, and $\mathrm{CH}_{4}$. Early results of this work were presented in Ruffio (2019). The current analysis is improved in several ways: we have gathered significantly more data on HR $8799 \mathrm{~d}$, the logarithm of spectral energy distribution of the molecular models were mistakenly used in Ruffio (2019) resulting in slightly lower $\mathrm{S} / \mathrm{N}$, and principal components are now included in the model. The $\mathrm{S} / \mathrm{N}$ curves are summarized in Figure 1. The detections are compared to samples of the noise in Figures 2 and 3 for $H$ band and $K$ band, respectively. The histograms of the noise are shown in Figure 4. The detection $\mathrm{S} / \mathrm{Ns}$ in $H$ band and $K$ band are given as a function of the number of principal components in Tables 1 and 2, demonstrating the gains brought by the improved forward model.

Here, we estimate the noise from the surrounding speckles, so the $\mathrm{S} / \mathrm{N}$ does not include sources of noise that are planet specific, such as additional photon noise or additional telluric residuals. This remains valid as long as the planet signal is negligible compared to starlight (i.e., speckles), which is likely true for HR $8799 \mathrm{c}$ and d, but could start to break at the large separation of HR 8799 b. Confusion, or crosstalk, between templates can also occur due to overlapping spectral lines, introducing systematics. The crosstalk between $\mathrm{H}_{2} \mathrm{O}$ and $\mathrm{CO}$ is minimal compared to the strength of their respective detection and therefore can be neglected. In other words, the detections of $\mathrm{H}_{2} \mathrm{O}$ and $\mathrm{CO}$ are far too strong to be explained by a crosstalk with another molecule. An illustration of all the crosstalks between templates can be found in Figure D.11 in Ruffio (2019). The situation is different for methane, there is no clear detection for any of the three planets, and the amplitude of the signal $(\mathrm{S} / \mathrm{N} \sim 5)$ is consistent with the amplitude of the crosstalk between $\mathrm{CH}_{4}$ and strong $\mathrm{H}_{2} \mathrm{O}$ and $\mathrm{CO}$ detections. In this case, the large $\mathrm{S} / \mathrm{N}$ value for $\mathrm{CH}_{4}$ and $\mathrm{HR} 8799$ b only signifies that the null hypothesis is rejected (i.e., there is indeed a planet at the considered location). In other words, the methane signal appears too weak to be confidently disentangled from the crosstalk and no detection can be claimed despite the apparent strength of the signal at the expected radial velocity of the planet.

$\mathrm{CCF}$-like analyses are interesting, because they provide an unambiguous and straightforward way to detect the molecules with the strongest signatures in the atmosphere of exoplanets. The estimation of molecular abundances or enrichment ratios will be addressed in Section 7.

\section{Radial Velocity}

In this section, we present the first RV measurements of HR $8799 \mathrm{~d}$, and we revisit the values presented in Ruffio et al. (2019) for HR $8799 \mathrm{~b}$ and $\mathrm{c}$ using an improved data model (Wilcomb et al. 2020). For each exposure in which the planet is detected, we compute the RV posterior according to Section 3.7 with an RV sampling from $-80 \mathrm{~km} \mathrm{~s}^{-1}$ to $+80 \mathrm{~km} \mathrm{~s}^{-1}$ with a step size of $0.4 \mathrm{~km} \mathrm{~s}^{-1}$. This range of RVs corresponds to an absolute spectral shift of two spectral bins in either direction for OSIRIS. The coarse sampling of $0.4 \mathrm{~km} \mathrm{~s}^{-1}$ is satisfactory for single exposure uncertainties, but it is later interpolated on a finer grid when combining exposures together. We use the same spectral templates as the one used for detecting the planets.

Figure 5 shows the nightly combined RVs of the three planets; they are also listed in Table 3 with existing literature values. The updated RVs for HR 8799 b and c are still consistent with Ruffio et al. (2019). Figure 6 shows the RV evolution as a function of the number of principal components used in the analysis. The median planets' RV variations over ten years (2010-2020) are relatively small based on the visual orbits for HR $8799 \mathrm{~b}$ and c: $\Delta R V_{\mathrm{b}}=0.1 \mathrm{~km} \mathrm{~s}^{-1}$ and $\Delta R V_{\mathrm{c}}=0.4 \mathrm{~km} \mathrm{~s}^{-1}$. The difference between 2015 and 2020 for HR $8799 \mathrm{~d}$ is $\Delta R V_{\mathrm{d}}=-0.4 \mathrm{~km} \mathrm{~s}^{-1}$. As a first analysis, we therefore compare the weighted mean RVs to the predictions from astrometry assuming coplanar and stable orbits (Wang et al. 2018b). We achieve subkilometer-per-second precision on the RV of three planets. A full orbital analysis using individual nightly RVs is performed in Section 6 assuming coplanar orbits.

\section{Orbital Analysis}

Following the same approach as in Ruffio et al. (2019), we fit for the orbits of HR 8799 b, c, and d, assuming the three planets are coplanar. We use the same modified branch from 


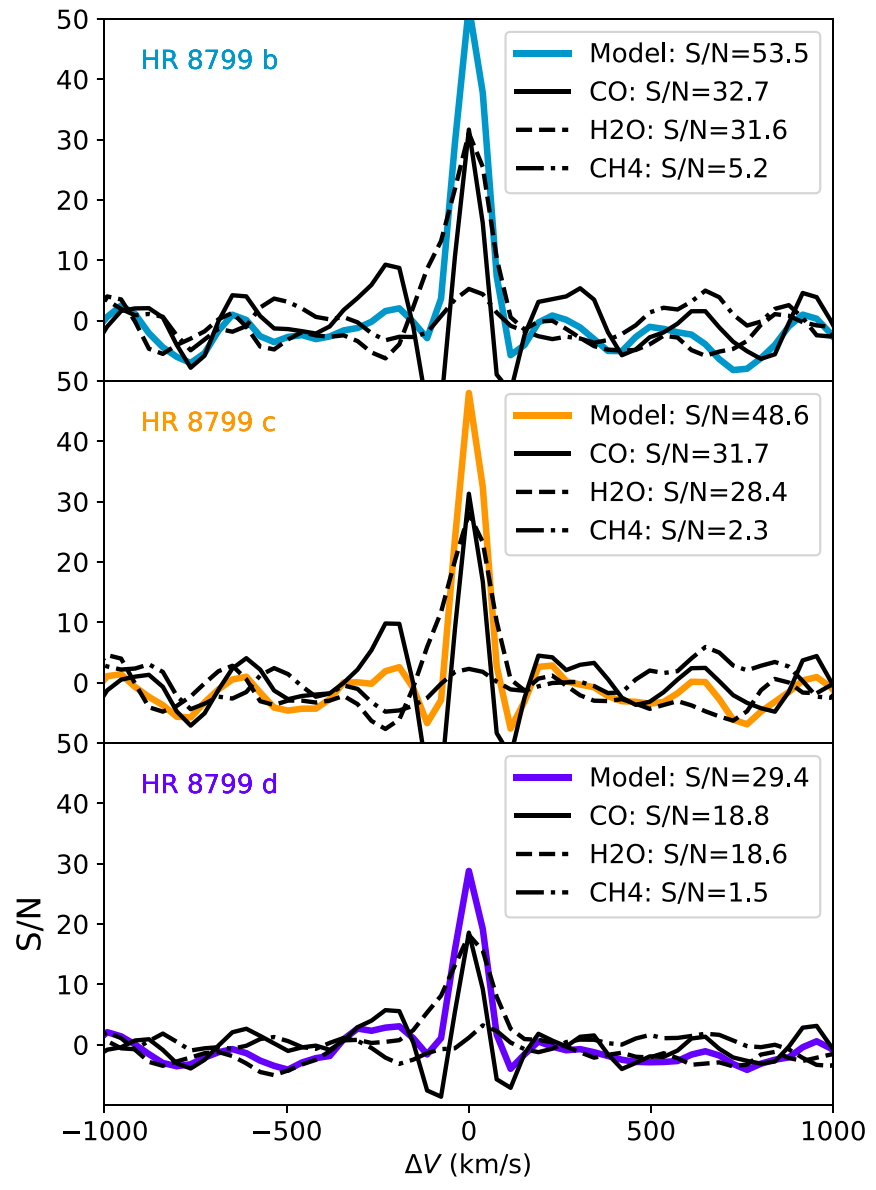

(a) $\mathrm{K}$ band

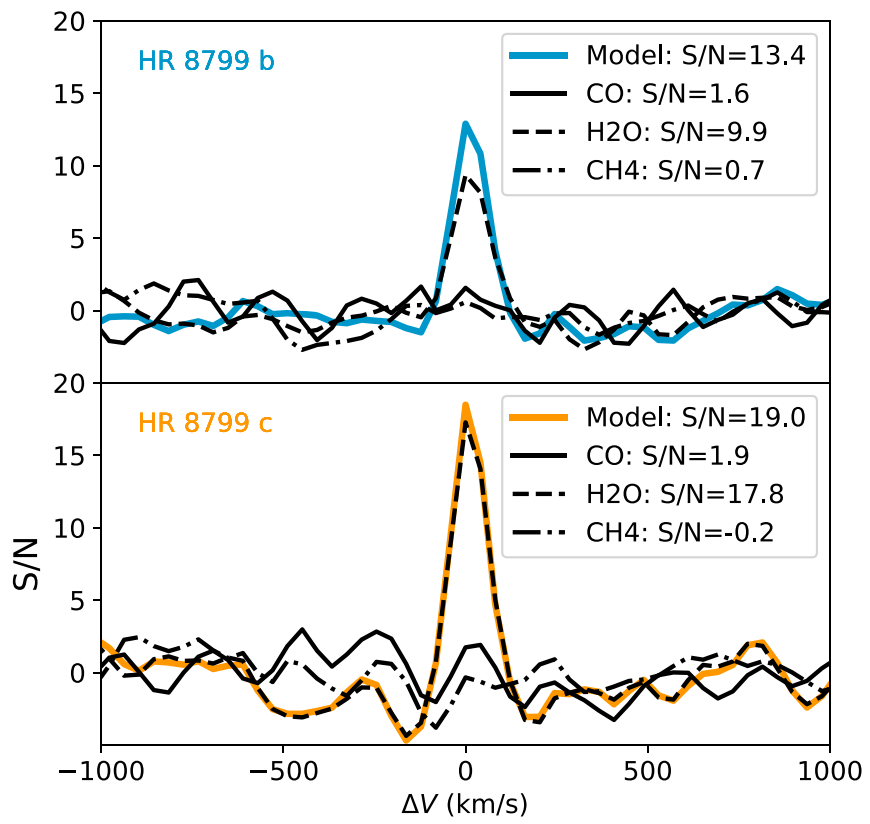

(b) $\mathrm{H}$ band

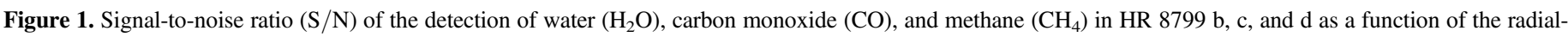

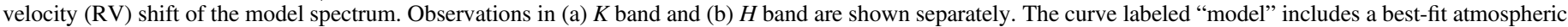

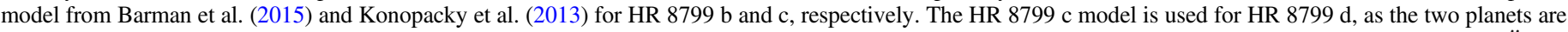

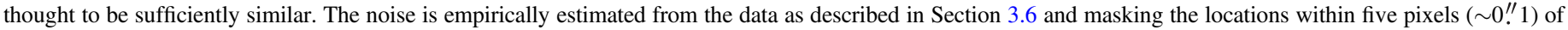
the companion.

the open source python package orbitize! (Blunt et al. 2019, 2020) with the parallel tempered implementation of the affine-invariant ensemble sampler for Markov chain Monte Carlo (ptemcee; Foreman-Mackey et al. 2013; Vousden et al. 2016). We compare the resulting orbital constraints when including both the relative astrometry and the planetary RV measurements to the astrometry only case. The data included in the fit are the RVs from Table 3, and the relative astrometry measurements from Keck/NIRC2 (Konopacky et al. 2016), and Gemini/GPI (Wang et al. 2018b). The priors for each parameter are listed in Table 4. We use 16 temperatures and 512 walkers, and we only save the position of the walkers every 50 steps. For the RV case, we ran the chains for 400,000 steps $\left(\approx 2 \times 10^{8}\right.$ samples in total), which includes a 200,000 step burn-in phase. The autocorrelation length is between 500 and 1500 steps depending on the parameters, which gives about 200 independent samples per walker and $\sim 100,000$ in total. When not including the RV, the chains were 200,000 steps including a 100,000-step burn-in phase.

Figure 7 shows 50 orbits randomly sampled from the posterior of the RV fit compared to the data points. The associated marginalized posteriors can be found in Figure 8 and the values of the estimated parameters are reported in Table 4. The covariance between the inclination and the longitude of ascending node for the orientation of the orbital plane, and the eccentricity and semimajor axis for the shape of the orbits are shown in Figure 9.

\section{Atmosphere Characterization}

\subsection{Atmospheric Model Grid}

We now measure the carbon-to-oxygen ratios $(\mathrm{C} / \mathrm{O})$ of $\mathrm{HR}$ 8799 b, c, and d using a grid of PHOENIX atmospheric models initially described in Barman et al. (2011a). The models use the updated methane line list from Yurchenko \& Tennyson (2014) and the optical opacities from Karkoschka \& Tomasko (2010), as in Barman et al. (2015). Disequilibrium chemistry is modeled by a coefficient of eddy diffusion, $K_{\mathrm{zz}}=10^{8} \mathrm{~cm}^{2} \mathrm{~s}^{-1}$. Clouds are included using the parameterized cloud model described in Barman et al. (2011a). The lower cloud boundary is determined assuming chemical equilibrium and the upper cloud boundary is modeled by an exponential decay starting at a specified pressure 


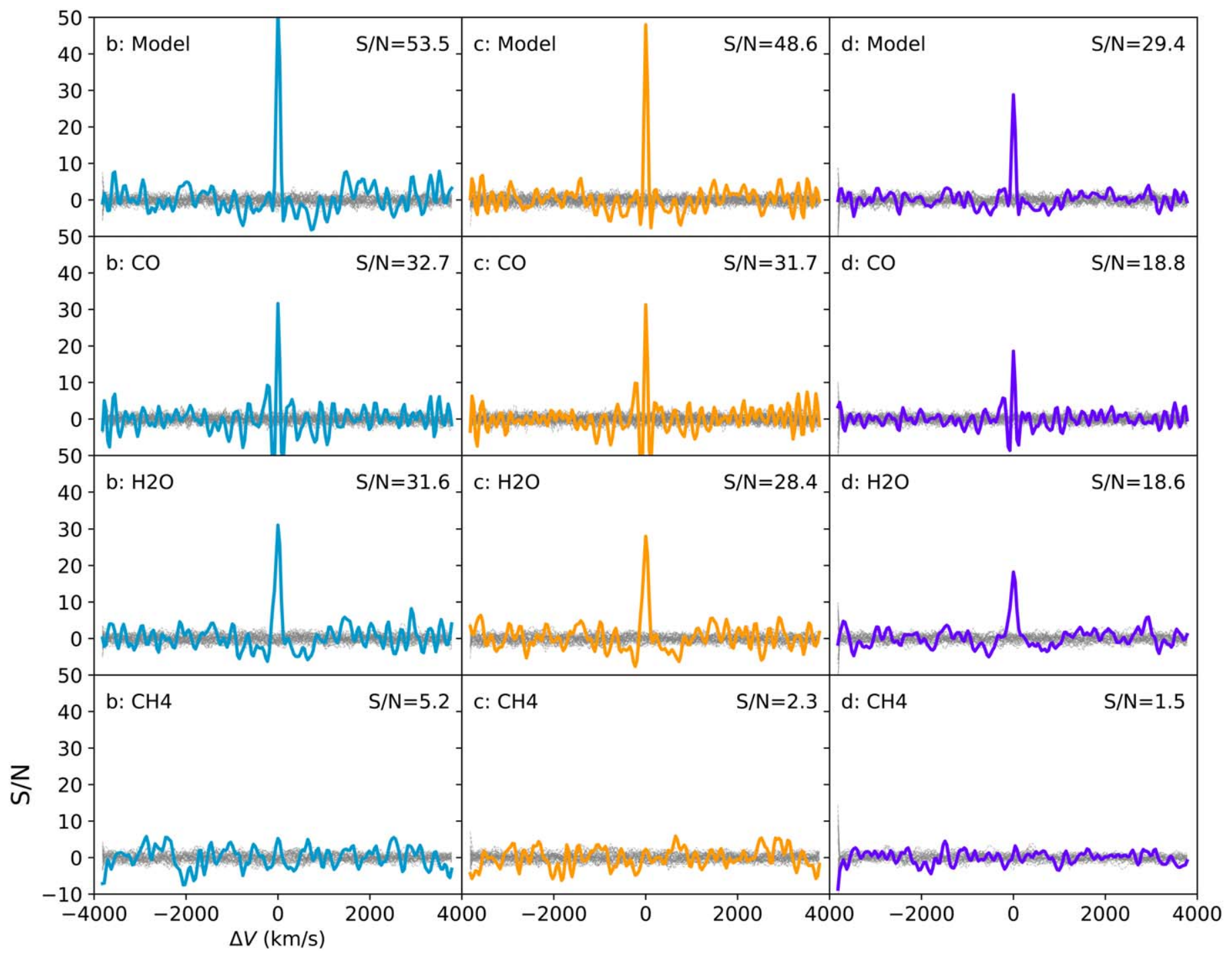

Figure 2. Similar to Figure 1 for $K$ band, but each curve is shown separately and compared to 50 samples of the noise (gray curves).

(Barman et al. 2015). The specified pressure is a free parameter, set to $4 \times 10^{6}$ dynes $\mathrm{cm}^{-2}$ in this work, that determines the thickness of the clouds, allowing for the models to have clouds that range from low opacity or cloudfree (COND) to high opacity (DUSTY; Allard et al. 2012). The particle size distribution is log-normal with a mean size chosen to be $5 \mu \mathrm{m}$. Due to the computational cost of generating the models, the oxygen and carbon abundances are not varied individually but using only the $\mathrm{C} / \mathrm{O}$ ratio. The $\mathrm{C} / \mathrm{O}$ traces the ratio of accreted solids to gas in the planet atmosphere, assuming it formed between the $\mathrm{CO}_{2}$ and $\mathrm{CO}$ snow lines (see Figure 10 in Öberg et al. 2011; Barman et al. 2015). In the original grid, temperature varies from $800 \mathrm{~K}$ to $1200 \mathrm{~K}$ in steps of $100 \mathrm{~K}$, surface gravity $\log \left(g /\left[1 \mathrm{~cm} \mathrm{~s}^{-2}\right]\right)$ varies from 3 to 4.5 in steps of 0.5 , and $\mathrm{C} / \mathrm{O}$ varies from 0.45 to 0.90 in steps of 0.05 . $\mathrm{C} / \mathrm{O}$ is defined as a ratio of number of molecules. The wavelength sampling of the models is $\delta \lambda \approx 5 \times 10^{-6} \mu \mathrm{m}(\lambda /$ $\delta \lambda \sim 426,000)$ compared to OSIRIS' pixels that have $\delta \lambda \approx$ $2.5 \times 10^{-4} \mu \mathrm{m}(\lambda / \delta \lambda \sim 8500)$. The $[\mathrm{C} / \mathrm{H}]$ and $[\mathrm{O} / \mathrm{H}]$ are given as a function of $\mathrm{C} / \mathrm{O}$ in Figure 10.

\subsection{Forward Modeling with OSIRIS}

We infer the four parameters $\left[T_{\text {eff }}, \log (g), \mathrm{C} / \mathrm{O}, \mathrm{RV}\right]$ according to Section 3.7 by linearly interpolating the models on a finer grid. The best-fit models are compared to the combined extracted spectra in Figure 11. These spectra are not used in the definition of the likelihood, but only used as visualization tool. They feature well-resolved $\mathrm{H}_{2} \mathrm{O}$ and $\mathrm{CO}$ spectral signatures. By defining the likelihood from the spectral cubes directly, hence avoiding the spectral extraction step, and jointly modeling the starlight and the planet, we prevent the potential biases caused by oversubtraction of the companion signal that are very common in high-contrast imaging (Marois et al. 2014; Pueyo 2016). Moreover, an interesting property of this framework is the marginalization over the starlight subtraction itself.

The different likelihoods for each exposures can be combined assuming statistical independence. In this case, there is no difference between posterior and likelihood because we are not introducing any special prior. We evaluate the consistency of the $\mathrm{C} / \mathrm{O}$ estimates by marginalizing the posterior for each exposure 


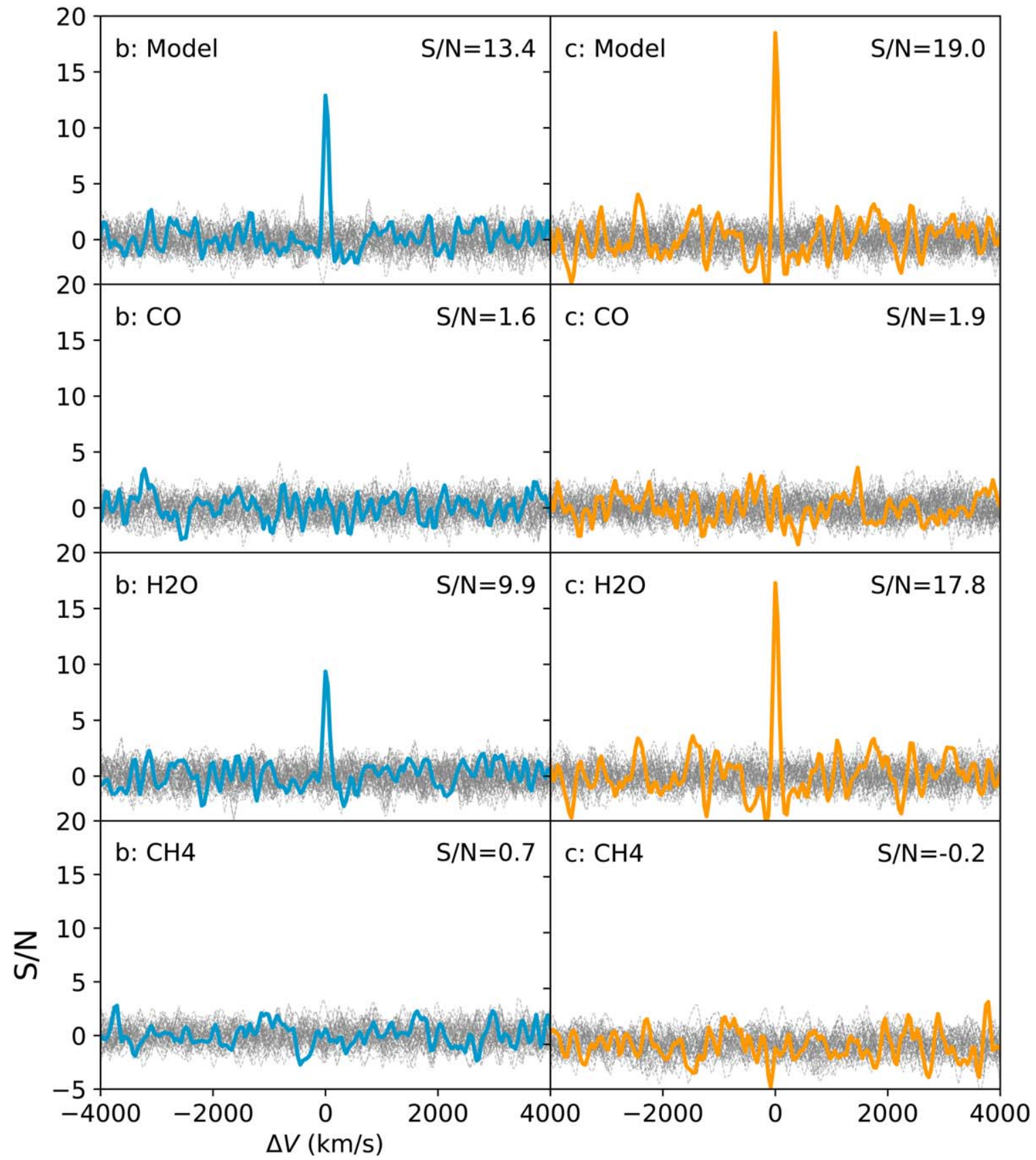

Figure 3. Similar to Figure 1 for $H$ band, but each curve is shown separately and compared to 50 samples of the noise (gray curves).

and the result is shown in Figure 12. The ability to test the modeling of the noise by fitting every exposure individually is one of the advantages of using a grid of models compared to the more computationally costly full retrieval. After marginalizing over the planetary $\mathrm{RV}$, the $\left[T_{\text {eff }}, \log (g), \mathrm{C} / \mathrm{O}\right]$ posteriors are shown in Figure 13 and the corner plots can be found in Appendix C.

The best-fit parameters corresponding to Figure 11 are summarized in Table 5. The combined $\mathrm{C} / \mathrm{O}$ estimates for $\mathrm{HR}$ $8799 \mathrm{~b}, \mathrm{c}$, and d are $0.572_{-0.007}^{+0.008}, 0.562_{-0.007}^{+0.003}$, and $0.55_{-0.03}^{+0.02}$ respectively. We recognize that these formal errors are highly underestimated and do not include the broader level of uncertainty surrounding their estimation due to imperfect data calibrations and the definition of the model grid. First, inferred temperature resulting from this fit is meeting the upper end of the grid at $1200 \mathrm{~K}$, which means that any covariance between temperature and $\mathrm{C} / \mathrm{O}$ would bias the values quoted previously. Simply extending the grid to include higher temperatures is not necessarily a solution as these values are thought to be unlikely in view of evolutionary models and the use of inaccurate temperatures can also bias the inference. As discussed in Konopacky et al. (2013) and Barman et al. (2015), these issues are known, and resolving them is outside the scope of this work. Second, another well-documented issue is the coarseness of the grid of atmospheric models (Czekala et al. 2015). The derived formal uncertainties are almost an order of magnitude smaller 


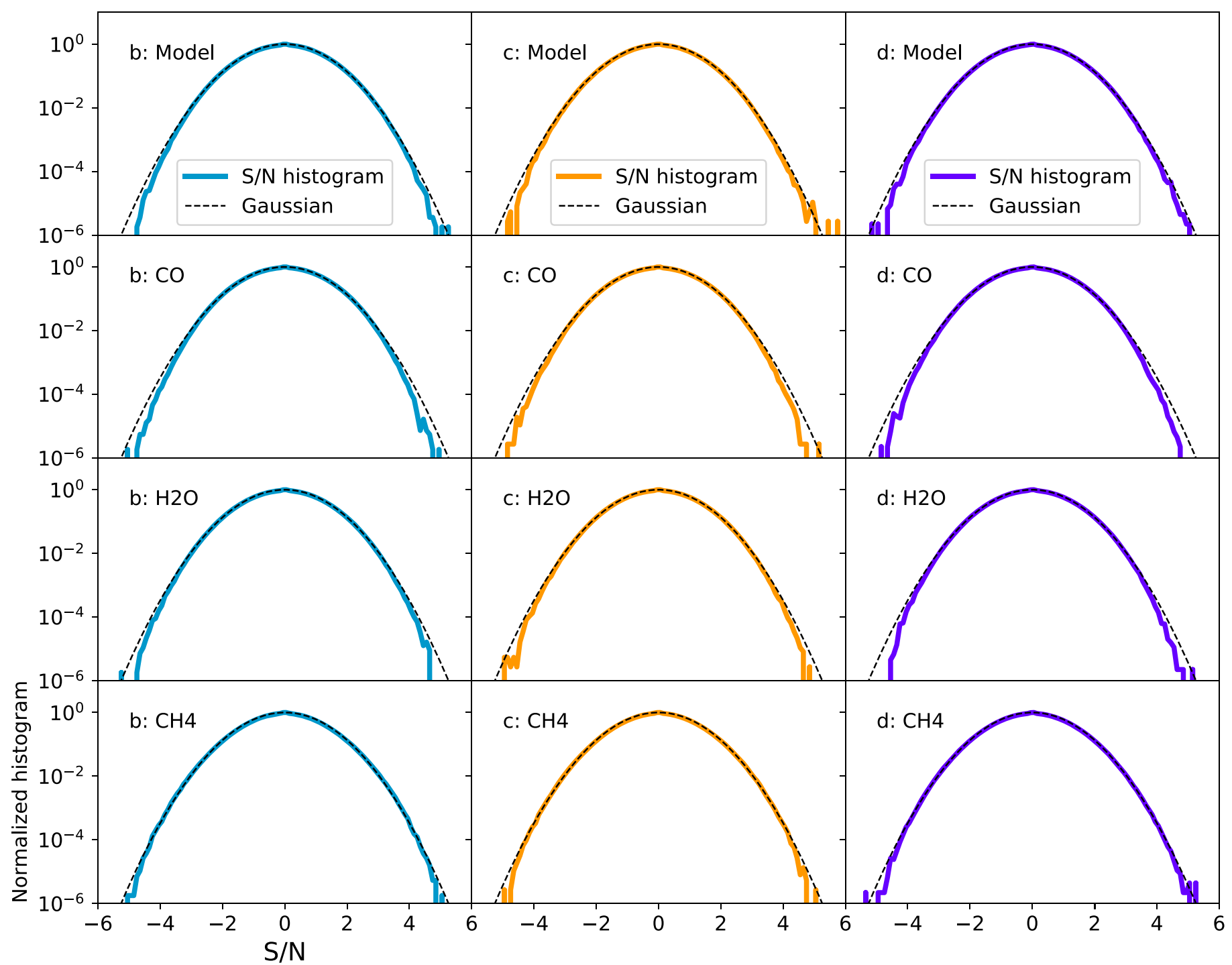

Figure 4. Histogram of the noise samples for each panel of Figure 2. The noise distribution is compared to a Gaussian normalized to a unit peak.

Table 1

$K$-band Detection S/N of Molecules as a Function of the Number of Principal Components Used in the Forward Model

\begin{tabular}{|c|c|c|c|c|c|c|c|c|c|}
\hline \multirow[b]{2}{*}{ \# PCs } & \multicolumn{3}{|c|}{ HR 8799 b } & \multicolumn{3}{|c|}{ HR 8799 c } & \multicolumn{3}{|c|}{ HR 8799 d } \\
\hline & 0 & 1 & 10 & 0 & 1 & 10 & 0 & 1 & 10 \\
\hline Model & 51.7 & 51.4 & 53.5 & 42.5 & 43.4 & 48.6 & 19.7 & 21.2 & 29.4 \\
\hline $\mathrm{CO}$ & 32 & 32 & 32.7 & 27.5 & 28 & 31.7 & 12.2 & 13.2 & 18.8 \\
\hline $\mathrm{H}_{2} \mathrm{O}$ & 29 & 30 & 31.6 & 23.7 & 24.5 & 28.4 & 12.5 & 13.1 & 18.6 \\
\hline $\mathrm{CH}_{4}$ & 5.5 & 5.1 & 5.2 & 1.7 & 2.1 & 2.3 & 2.3 & 1.9 & 1.5 \\
\hline
\end{tabular}

Table 2

Same as Table 1 for $H$ Band

\begin{tabular}{llcccccc}
\hline \hline & \multicolumn{3}{c}{ HR 8799 b } & & \multicolumn{3}{c}{ HR 8799 c } \\
\cline { 2 - 4 } \cline { 6 - 8 } \# PCs & 0 & 1 & 10 & & 0 & 1 & 10 \\
\hline Model & 12.2 & 13.2 & 13.4 & & 15.3 & 15.8 & 19 \\
$\mathrm{CO}$ & 1.4 & 1.4 & 1.6 & & 1.9 & 1.3 & 1.2 \\
$\mathrm{H}_{2} \mathrm{O}$ & 9.0 & 9.6 & 9.9 & & 14.5 & 14.9 & 17.8 \\
$\mathrm{CH}_{4}$ & 0.4 & 0.7 & 0.7 & & -0.3 & -0.4 & -0.2 \\
\hline
\end{tabular}

that the step size in each dimension $\left(\Delta T_{\text {eff }}=100 \mathrm{~K}, \Delta \log (g)=\right.$ $0.5, \Delta \mathrm{C} / \mathrm{O}=0.05)$. The local minima in the $\mathrm{C} / \mathrm{O}$ posteriors of HR $8799 \mathrm{~b}$ and other kinks in Figure 13 are not real and match the sampling of grid. In the following section, we fit the same grid of models to published photometry and low-resolution spectra as an independent way to evaluate the consistency of the posteriors. While acknowledging these issues, we can conclude that the three planets appear to have similar $\mathrm{C} / \mathrm{O}$ without large deviations from the stellar value. 


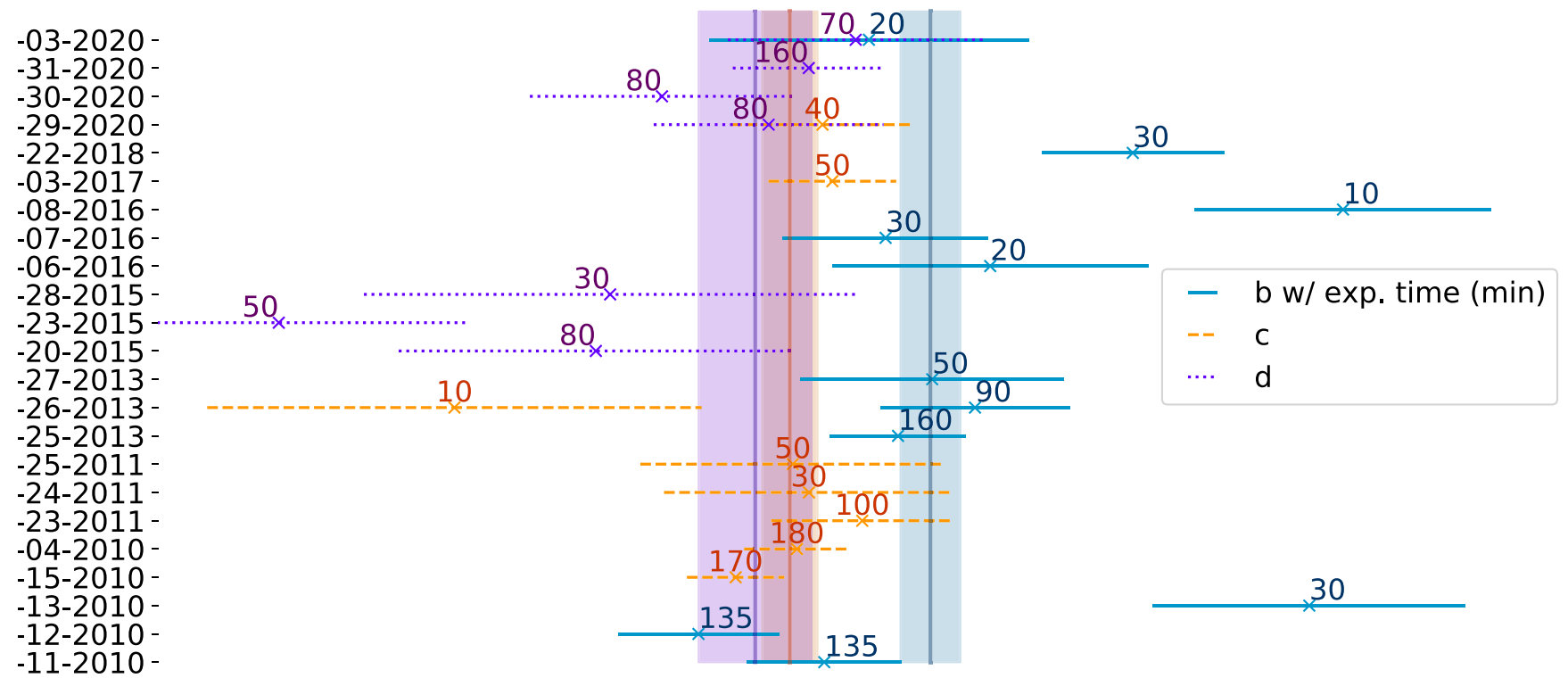

\begin{tabular}{|c|c|c|c|c|c|c|}
\hline-20.0 & -17.5 & -15.0 & -12.5 & $\begin{array}{c}-10.0 \\
\operatorname{RV}(\mathrm{km} / \mathrm{s})\end{array}$ & -7.5 & 0.0 \\
\hline
\end{tabular}
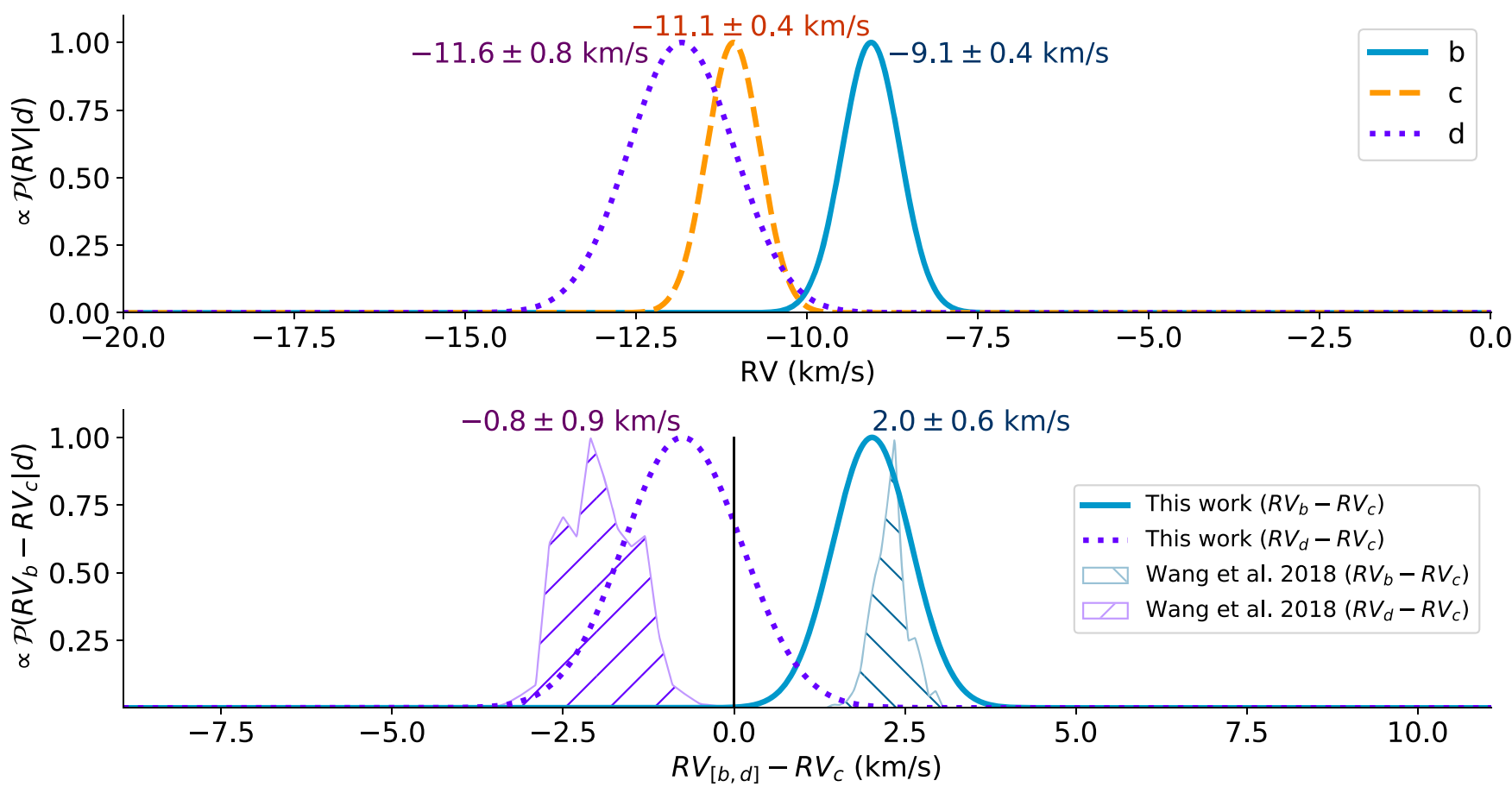

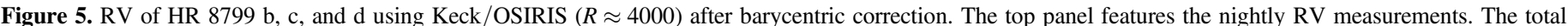

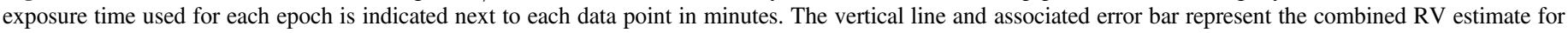

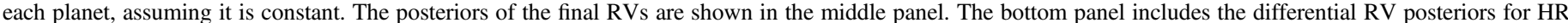

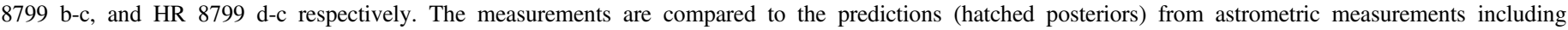

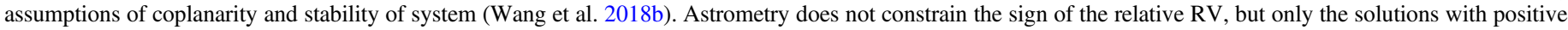
$\mathrm{RV}_{\mathrm{b}}-\mathrm{RV}_{\mathrm{c}}$ are shown here.

\subsection{Fitting the Spectral Energy Distribution}

We can compare the atmospheric model fits from OSIRIS to lower resolution published data. Figure 13 contains the marginalized posteriors for temperature, surface gravity, and
$\mathrm{C} / \mathrm{O}$ for the three planets as a function of the data set used: photometry, low-resolution spectra, and moderate resolution with OSIRIS. The best-fit models are compared to the data in Figure 14. The different fits are described below. 
Table 3

Barycentric Corrected Radial Velocities of HR 8799 b, c, and d Measured with Keck/OSIRIS

\begin{tabular}{|c|c|c|c|c|c|}
\hline Planet & Date & MJD & $\begin{array}{l}\mathrm{RV}\left(\mathrm{km} \mathrm{s}^{-1} \text { ) }\right. \\
\text { (this Work) }\end{array}$ & $\begin{array}{c}\left.\text { RV (km s }{ }^{-1}\right) \\
\text { Ruffio et al. (2019) }\end{array}$ & $\begin{array}{c}\text { Time } \\
\text { (minutes) }\end{array}$ \\
\hline \multirow[t]{11}{*}{$\mathrm{b}$} & 2010-07-11 & 55388.55 & $-10.6 \pm 1.1$ & $-10.2 \pm 1.2$ & 135 \\
\hline & 2010-07-12 & 55389.54 & $-12.4 \pm 1.1$ & $-10.1 \pm 1.1$ & 135 \\
\hline & $2010-07-13$ & 55390.62 & $-3.7 \pm 2.2$ & $-4.0 \pm 2.4$ & 30 \\
\hline & $2013-07-25$ & 56498.55 & $-9.5 \pm 1.0$ & $-8.9 \pm 1.0$ & 160 \\
\hline & $2013-07-26$ & 56499.50 & $-8.4 \pm 1.3$ & $-9.4 \pm 1.4$ & 90 \\
\hline & $2013-07-27$ & 56500.51 & $-9.1 \pm 1.9$ & $-6.5 \pm 2.3$ & 50 \\
\hline & 2016-11-06 & 57698.32 & $-8.2 \pm 2.2$ & $-10.8 \pm 2.2$ & 20 \\
\hline & 2016-11-07 & 57699.32 & $-9.7 \pm 1.5$ & $-11.8 \pm 1.5$ & 30 \\
\hline & 2016-11-08 & 57700.34 & $-3.2 \pm 2.1$ & & 10 \\
\hline & $2018-07-22$ & 58321.52 & $-6.2 \pm 1.3$ & $-6.7 \pm 1.3$ & 30 \\
\hline & $2020-08-03$ & 59064.46 & $-9.9 \pm 2.3$ & & 20 \\
\hline \multirow[t]{8}{*}{$\mathrm{c}$} & $2010-07-15$ & 55392.55 & $-11.8 \pm 0.7$ & $-11.8 \pm 0.7$ & 170 \\
\hline & 2010-11-04 & 55504.31 & $-11.0 \pm 0.7$ & $-11.5 \pm 0.8$ & 180 \\
\hline & 2011-07-23 & 55765.54 & $-10.0 \pm 1.3$ & $-10.8 \pm 1.3$ & 100 \\
\hline & 2011-07-24 & 55766.56 & $-10.8 \pm 2.1$ & & 30 \\
\hline & 2011-07-25 & 55767.57 & $-11.0 \pm 2.2$ & $-18.8 \pm 5.7$ & 50 \\
\hline & $2013-07-26$ & 56499.58 & $-15.8 \pm 3.5$ & & 10 \\
\hline & $2017-11-03$ & 58060.25 & $-10.5 \pm 0.9$ & & 50 \\
\hline & $2020-07-29$ & 59059.50 & $-10.6 \pm 1.3$ & & 40 \\
\hline \multirow[t]{8}{*}{$\mathrm{d}$} & $2015-07-20$ & 57223.51 & $-13.8 \pm 2.8$ & & 80 \\
\hline & $2015-07-23$ & 57226.52 & $-18.3 \pm 2.7$ & & 50 \\
\hline & $2015-08-28$ & 57262.54 & $-13.6 \pm 3.5$ & & 30 \\
\hline & $2020-07-29$ & 59059.59 & $-11.4 \pm 1.6$ & & 80 \\
\hline & 2020-07-30 & 59060.56 & $-12.9 \pm 1.9$ & & 80 \\
\hline & 2020-07-31 & 59061.54 & $-10.8 \pm 1.1$ & & 160 \\
\hline & $2020-08-03$ & 59064.55 & $-10.1 \pm 1.8$ & & 70 \\
\hline & & & & Wang et al. (2018a) & \\
\hline c & 2016-2017 & & & $-8.9 \pm 2.5$ & $15.6 \mathrm{hr}$ \\
\hline HR 8799 & 2016-2017 & & & $-10.9 \pm 0.5$ & $15.6 \mathrm{hr}$ \\
\hline
\end{tabular}

Note. Literature RVs for the planets and the host star are given for comparison.

First, we fit the photometric data points from the literature and summarized in Table 6 . The $\mathrm{C} / \mathrm{O}$ model grid used in this work is limited to $H$ and $K$ bands, which is why we cannot include the existing 3-5 $\mu \mathrm{m}$ photometry in this analysis. The conversion between absolute magnitudes and flux is done using each instrument filter ${ }^{15}$ and a reference Vega spectrum ${ }^{16}$ (Bohlin et al. 2014). Variations of the atmosphere water content and transmission is assumed negligible and was not accounted for. We generated from the high-resolution atmospheric models a grid of photometric points, which was then linearly interpolated on a finer grid. The fluxes were converted to magnitudes and then compared to the data with a $\chi^{2}$. In the absence of priors, the posterior is equal to the likelihood $\left(\propto \exp -\chi^{2} / 2\right)$.

Then, for the low-resolution spectra, we fit the $H$ - $(R=46), K 1$ $(R=66)$, and $K 2$-band $(R=79)$ GPI spectra from Greenbaum et al. (2018) for HR $8799 \mathrm{c}$ and $\mathrm{d}$, and the $H$-band spectrum ( $R=60)$ from Barman et al. (2011a) combined with a smoothed $(R=200)$ OSIRIS spectrum from Barman et al. (2015). The GPI linespread function is oversampled, so we are only using a fourth of the data points to limit possible issues related to correlation. We first convolved the model to match the sampling and resolution of the low-resolution spectra. The broadened model grid was then

\footnotetext{
15 http://svo2.cab.inta-csic.es/theory/fps/

16 https://www.stsci.edu/hst/instrumentation/reference-data-for-calibrationand-tools/astronomical-catalogs/calspec
}

linearly interpolated and a $\chi^{2}$ computed over a more finely sampled grid. We use a similar approach in the case of the photometric data points. The atmospheric models are only defined over $H+K$ band so we cannot include existing observations at shorter and longer wavelength. The results of this analysis of the photometry and low-resolution spectra compared to the higherresolution fits are discussed in Section 8.3.

\section{Discussion}

\subsection{Molecule Detection}

We used a method similar to cross correlation analysis to look for $\mathrm{H}_{2} \mathrm{O}, \mathrm{CO}$, and $\mathrm{CH}_{4}$ in the atmosphere of $\mathrm{HR} 8799 \mathrm{~b}, \mathrm{c}$, and $\mathrm{d} . \mathrm{H}_{2} \mathrm{O}$ and $\mathrm{CO}$ are detected in all three planets and these detection are at a higher $\mathrm{S} / \mathrm{N}$ compared to past analysis of the same data for HR 8799 b and c (Konopacky et al. 2013; Barman et al. 2015; Petit dit de la Roche et al. 2018). As expected, only $\mathrm{H}_{2} \mathrm{O}$ is detected in $\mathrm{H}$-band spectra. Significant efforts were made to limit the effects of potential systematics such as imperfect telluric correction (see Section 4).

Detecting and measuring the abundance of methane as a function of planet mass, age, and temperature is important to constrain nonequilibrium chemistry resulting from atmospheric mixing. The mixing efficiency is parametrized by the coefficient of eddy diffusion $K_{\mathrm{zz}}$. For HR 8799-like planets, an efficient mixing will result in the dissociation of methane in the hotter layer 
Table 4

Estimated Orbital Parameters from Joint Coplanar Fit of HR 8799 b, c, and d Using Planetary RVs

\begin{tabular}{|c|c|c|c|c|}
\hline \multirow{2}{*}{ Parameters } & \multicolumn{3}{|c|}{ HR 8799} & \multirow{2}{*}{ Priors } \\
\hline & $\mathrm{b}$ & $\mathrm{c}$ & $\mathrm{d}$ & \\
\hline$a(\mathrm{au})$ & $64.6_{-3.4}^{+3.7}$ & $40.9_{-1.8}^{+1.2}$ & $24.4_{-1.0}^{+0.7}$ & Inverse; $1 / a$ with $a \in\left[10^{-3}, 10^{7}\right]$ \\
\hline$\omega$ & $135_{-30}^{\circ+21}$ & $56_{-30}^{+249}$ & $>322^{\circ}$ & Uniform; $\omega \in[0,360]^{\circ}$ \\
\hline$\tau$ & $0.42_{-0.04}^{+0.06}$ & $0.35_{-0.19}^{+0.07}$ & $0.59_{-0.02}^{+0.03}$ & Uniform; $\tau \in[0,1]$ \\
\hline \multirow{2}{*}{$\begin{array}{l}i \\
\Omega\end{array}$} & & $19.2_{-4.5}^{+4.6}$ & & geometric ; $\sin (i)$ with $i \in[0,180]^{\circ}$ \\
\hline & & & & $\Omega \in[0,180]^{\circ}$ otherwise \\
\hline \multirow{3}{*}{$\begin{array}{l}\text { Parallax } \\
M_{\text {tot }} \\
\mathrm{RV}_{\star}\end{array}$} & & $24.2_{-0.1}^{+0.08} \mathrm{mas}$ & & Gaussian; $24.22 \pm 0.09$ mas \\
\hline & & $1.43_{-0.2}^{0.33} M_{\odot}$ & & Gaussian; $1.52 \pm 0.15 M_{\odot}$ \\
\hline & & $-10.5_{-0.2}^{+0.3} \mathrm{~km} \mathrm{~s}^{-1}$ & & Gaussian; $-12.6 \pm 1.4 \mathrm{~km} \mathrm{~s}^{-1}$ \\
\hline
\end{tabular}

Note. The error bars and upper/lower limits represent the $68 \%$ confidence interval. The last five parameters are shared by the two planets. The parallax, stellar mass, and stellar RV priors are respectively defined from Gaia Collaboration (2018), Baines et al. (2012), and Gontcharov (2006).

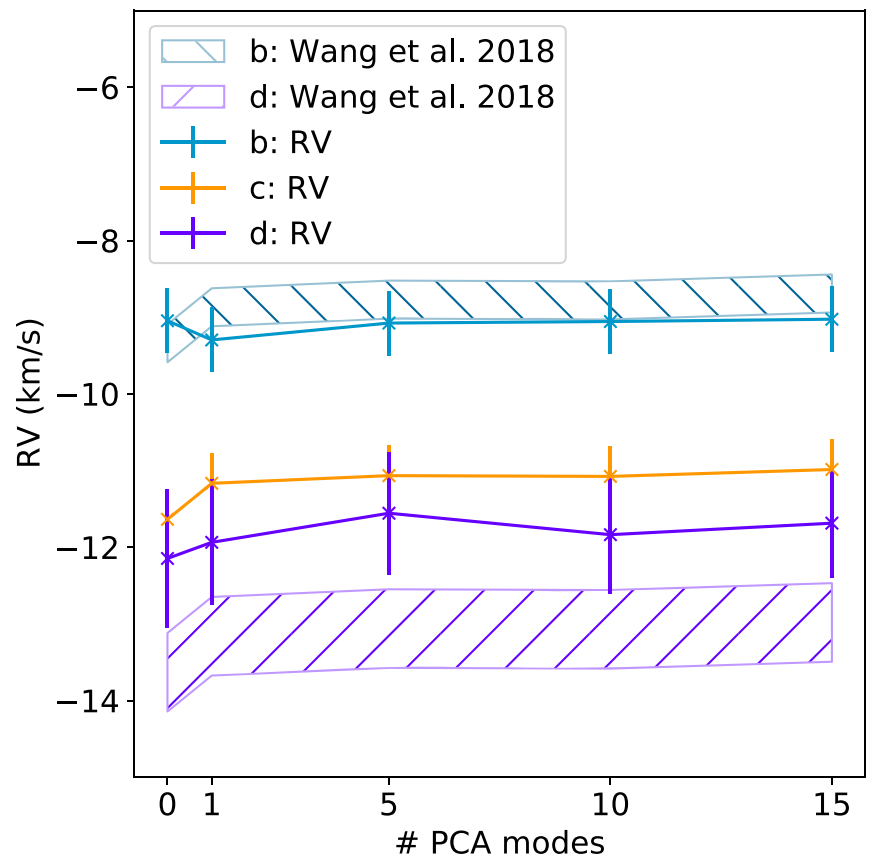

Figure 6. Combined radial velocities of HR 8799 b, c, and d as a function of the number of principal components used in the forward model of the data. As a reference, we show the RV predictions for $\mathrm{b}$ and $\mathrm{d}$ (hatched contours) by adding the predicted differential $\mathrm{RV}\left(\mathrm{RV}_{\mathrm{b}}-\mathrm{RV}_{\mathrm{c}}\right.$ or $\left.\mathrm{RV}_{\mathrm{d}}-\mathrm{RV}_{\mathrm{c}}\right)$ calculated from visual orbits (Wang et al. 2018b) to the measured $R_{\mathrm{c}}$.

of the atmosphere, decreasing its overall abundance. A detection of methane in HR $8799 \mathrm{~b}$ was presented in Barman et al. (2015) based on cross-correlation analysis and visible lines matching those of methane. A weak absorption band consistent with methane was also identified in a low-resolution $K$-band OSIRIS spectrum in Barman et al. (2011a). Using only a subset of this data set, Petit dit de la Roche et al. (2018) performed an independent cross correlation analysis that did not produce a similar detection of methane. They proposed that an imperfect telluric correction could explain the detection in the original work. To verify this hypothesis, we performed a new analysis of the results presented in Barman et al. (2015) that differed only by the telluric correction. Instead of computing the telluric transmission from the standard star observations, it is computed from the science spectral cubes themselves using the speckles from HR 8799A to account for telluric variability during the night. As shown in Appendix D, the methane signal in the CCF not only remains but it is significantly stronger when compared to Barman et al. (2015). In the present work and using a slightly different kind of analysis, we were also unable to produce a strong $\mathrm{CCF}$ detection of $\mathrm{CH}_{4}$ while including more observations. While the strength of the methane signal in the different CCF analyses is puzzling, we estimate that the lack of strong CCF detection of methane in this work is not inconsistent with the derived methane abundance from Barman et al. (2015) within the uncertainty. The abundance of methane derived in Barman et al. (2015; mole fraction between $10^{-5.06}$ and $10^{-5.85}$ ) suggested an eddy diffusion coefficient of $\log \left(K_{\mathrm{zz}}\right) \approx 7$. If the methane abundance was overestimated, a larger value of $K_{\mathrm{zz}}$ would be expected. A complete reanalysis of the individual molecular abundances of $\mathrm{H}_{2} \mathrm{O}$, CO, and $\mathrm{CH}_{4}$, as performed in Barman et al. (2015), is not within the scope of this work. The newly commissioned Keck Planet Imager and Characterizer (KPIC; $R \approx 37,500$; Mawet et al. 2017) instrument is a promising avenue to resolve this issue thanks to its order of magnitude higher spectral resolution.

\subsection{Radial Velocity and Orbits}

In Ruffio et al. (2019), we measured the RV of the outer two planets of the system. Here, we measured the RV of HR 8799 $\mathrm{d}$, the next-closest planet to the star. Similar to Wilcomb et al. (2020), we showed that imperfect telluric correction can result in significant RV systematics, which we corrected by included principal components of the residuals in the modeling of the data. The measurements from Ruffio et al. (2019) for HR 8799 $\mathrm{b}$ and $\mathrm{c}$ were also updated with the new model.

The inferred RVs for the three planets remain consistent with the astrometric measurements and assumptions of coplanarity and stability of the system (Wang et al. 2018b). We used the new RV to revisit the orbit analysis from Ruffio et al. (2019) while extending it to HR 8799 b, c, and d instead of HR 8799 b and c only. The three planets are assumed to be coplanar to speed up convergence. The past analysis already showed how the RV measurements of $b$ and $c$ ruled out the family of orbits with the longitude of ascending node $\left(\Omega \sim 280^{\circ}\right)$. The new RV measurement of HR $8799 \mathrm{~d}$ confirms this result, but it is otherwise providing marginal additional constraints on the other orbital parameters. Better constraining the eccentricity 

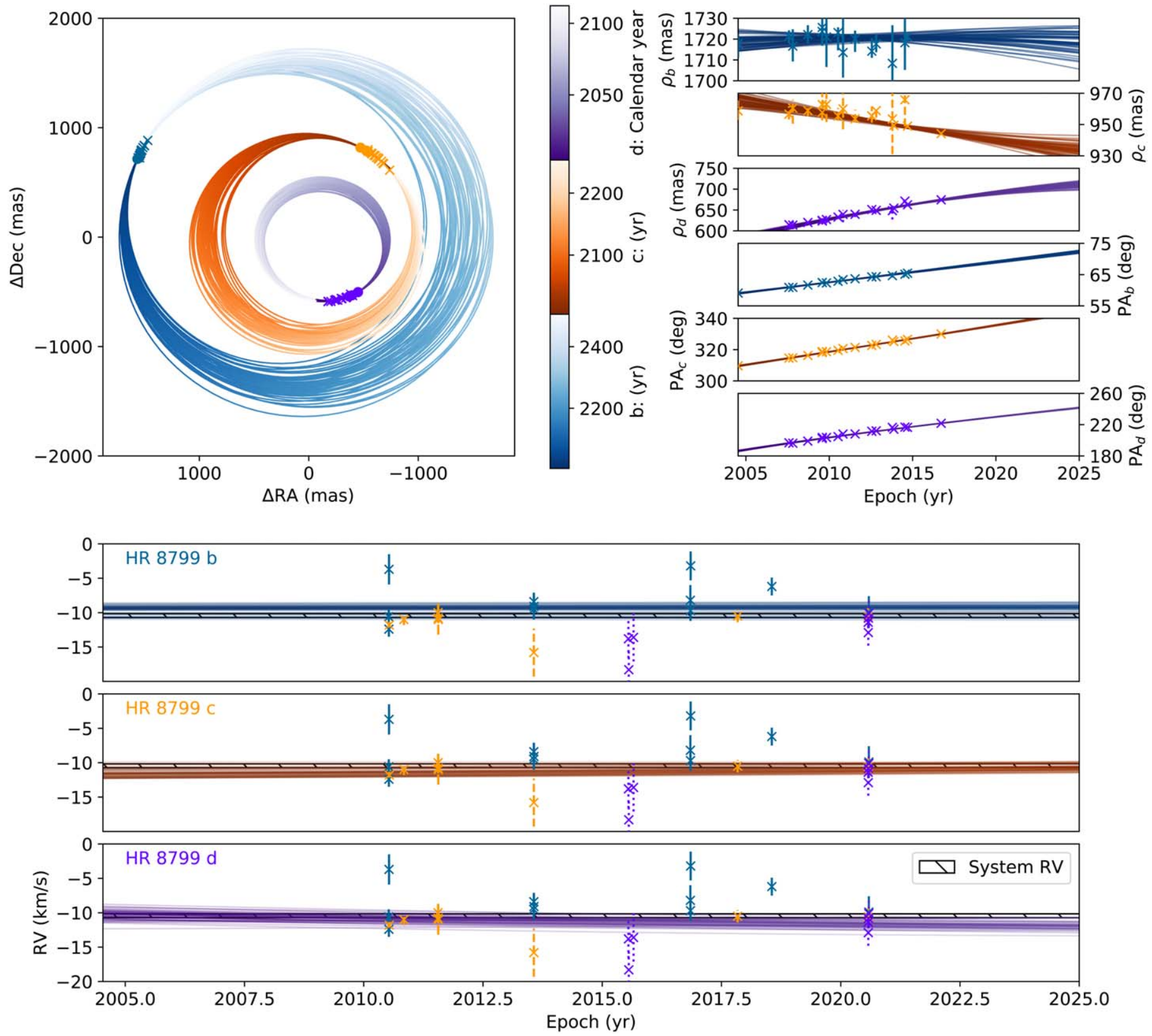

Figure 7. Orbits of HR 8799 b (blue), c (orange), and d (purple), randomly sampled from their posterior. The three planets are here assumed to be coplanar and the orbital fit include the RV measurements of the planets. From top to bottom on the upper right, the panels show the separation of the planets (b to d), and their position angle. The error bars were converted from R.A. and decl. to separation and position angle using a Monte Carlo approach. The bottom three panels are the RV measurement compared to the sample orbits (b to d).

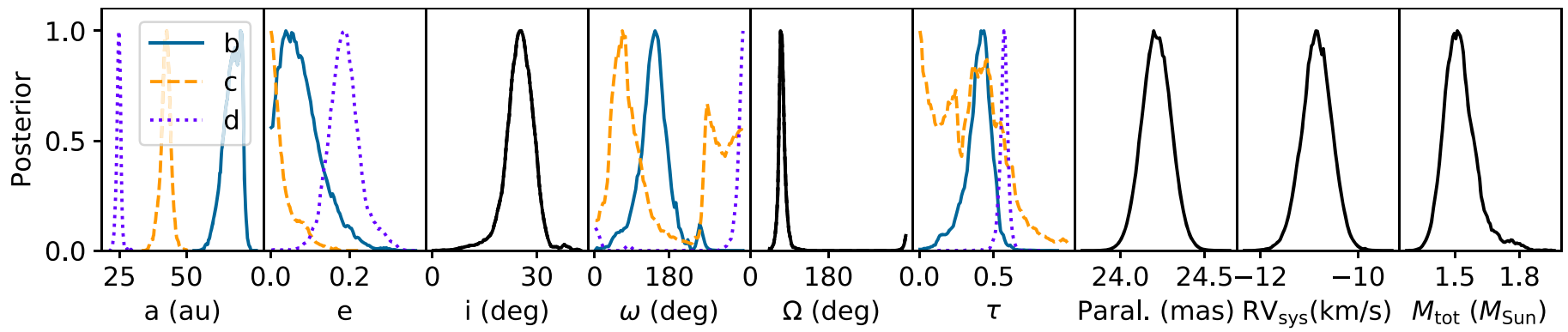

Figure 8. Marginalized posteriors of the orbital parameters from the joint coplanar fit of HR 8799 b, c, and d using planetary RVs. 


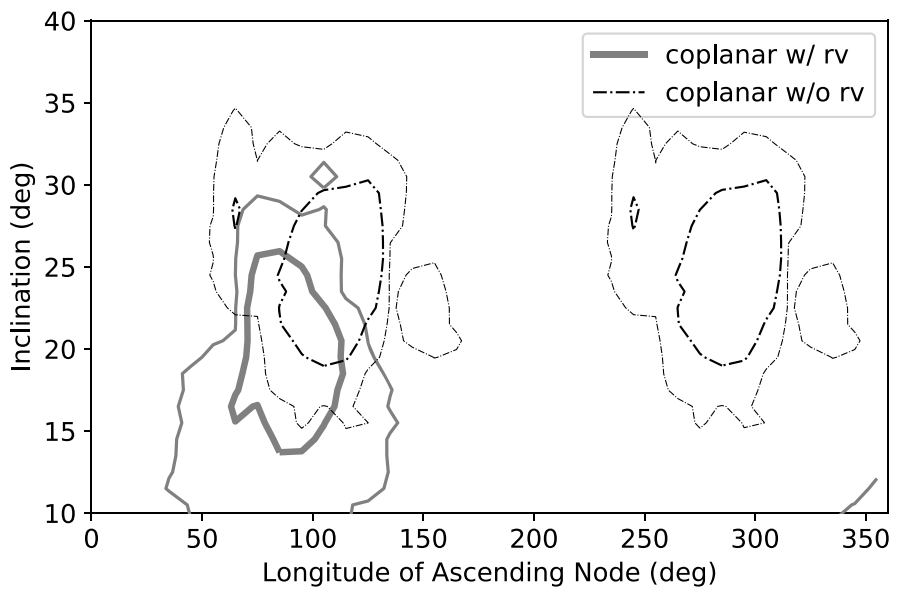

(a) Orbital plane Orientation

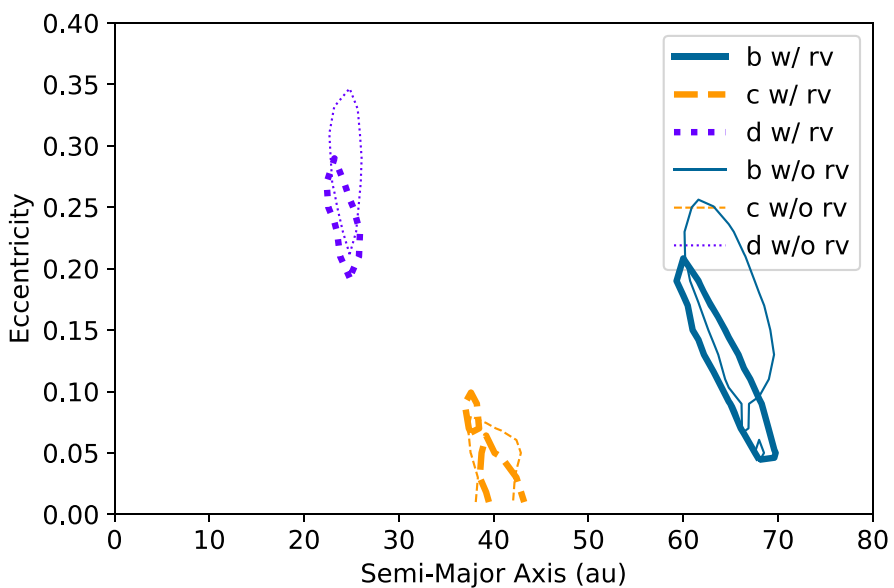

(b) Shape of the orbits

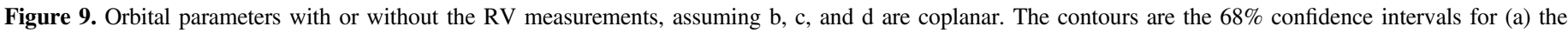

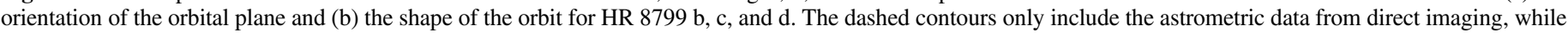

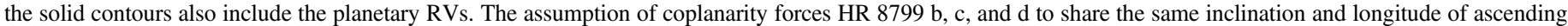

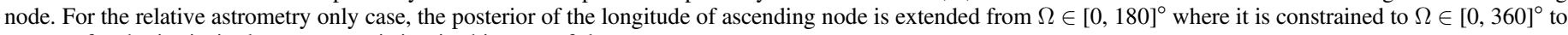
account for the intrinsic degeneracy existing in this type of data.

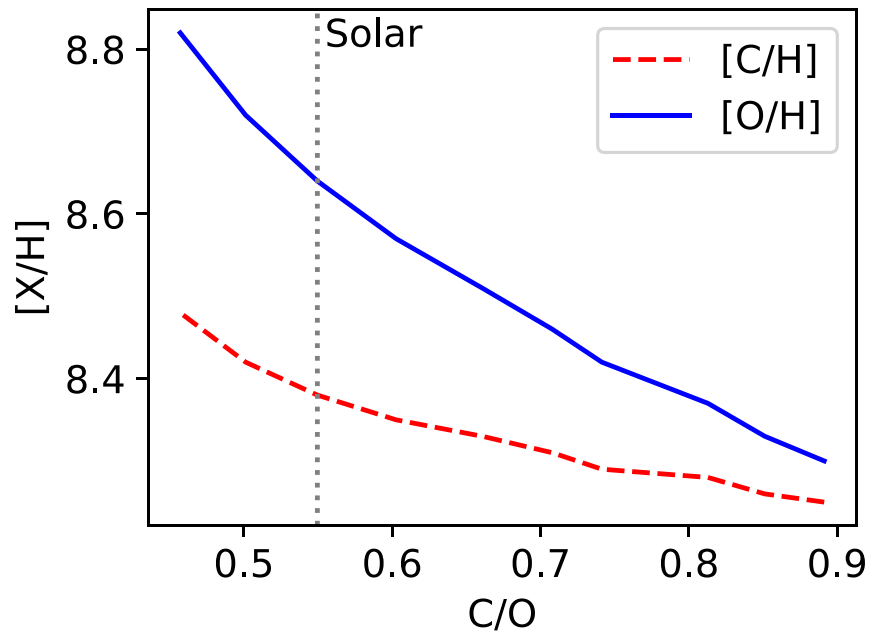

Figure 10. Log abundance by number of carbon and oxygen relative to hydrogen for the atmospheric model grid used in this work.

is important as a non-zero eccentricity while in resonance could point to the fact the planets migrated while in resonance (Wang et al. 2018b). Also differences in the eccentricity distribution of planets and brown dwarf could highlight different formation processes (Bowler et al. 2020). Planetary RVs are expected to be comparatively more powerful in cases for which the visual orbits are not as well constrained as HR 8799. The RV from Table 3 can be used to better constrain the mutual inclination of the HR 8799 planets, but this analysis is left for future work.

Measuring the RV of several components of a multiplanet system is interesting, because it removes the need for a precise RV measurement of the host star. The latter is often challenging as most stars orbited by directly imaged planets are young, sometimes variable, rapidly rotating, and early type stars. Snellen et al. (2014) and Wilcomb et al. (2020) are two examples in which the relative $\mathrm{RV}$ measurement of the planet is limited by our knowledge of the absolute RV of the star. In the near future, higher-resolution spectrographs such as KPIC should provide more precise planetary RVs. These measurements could be combined with extremely precise astrometric measurements such as the ones acquired by
GRAVITY for HR 8799 e (Gravity Collaboration et al. 2019). Altogether, these would improve our knowledge of the orbits of the system. These results are also important to demonstrate the feasibility of measuring RVs of planetary mass companions at high contrast. Such RV measurements have been proposed as a way to detect moons in the future (Vanderburg et al. 2018).

\subsection{Combining Data with Different Spectral Resolutions}

Combining low- and high-resolution spectra is a hallmark of the study of exoplanet atmospheres (Brogi \& Line 2019; Gravity Collaboration et al. 2020; Mollière et al. 2020; Wang et al. 2020), but developing models that can consistently fit both has proven to be challenging. For example, Wang et al. (2020) highlighted the difficulty in combining spectra by presenting separate and combined analyses of different instruments: GPI ( $H, R=46 ; \mathrm{K}, R=66-79)$, CHARIS (JHK, $R=20)$ and an OSIRIS spectrum binned down to $R=1000$ to characterize HR $8799 \mathrm{c}$. The prerequisite to combine spectra with different resolutions is to ensure that the posteriors from fitting them individually are consistent which each other. When jointly fitting the different resolutions, Wang et al. (2020) used a strong prior to fix the planet temperature and radius and to yield a planet mass for HR 8799 c consistent with the dynamical stability of the planetary system. Fixing a subset of parameters such as the temperature or surface gravity to better match the spectral energy distribution or cooling tracks of the planet is common practice. Zhang et al. (2020) also highlight the difficulty to retrieve surface gravity for brown dwarfs. However, this does not resolve the underlying inconsistencies of the model and the data, which therefore remains subject to systematics that are not well characterized.

The highlights of this analysis compare to past work are the uniform analysis of the three planets, and the higher spectral resolution from OSIRIS. The native resolution of OSIRIS $(R=4000)$ resolves the molecular lines of $\mathrm{H}_{2} \mathrm{O}$ and $\mathrm{CO}$, the dominant $\mathrm{C}$ and $\mathrm{O}$ carriers, which can unambiguously constrain $\mathrm{C} / \mathrm{O}$. For example, $\mathrm{CO}$ lines can be visually resolved in the $2.29 \mu \mathrm{m}$ CO band head for the three planets in the high $\mathrm{S} / \mathrm{N}$ spectra shown in Figure 11; this is a first for HR 8799 d. 


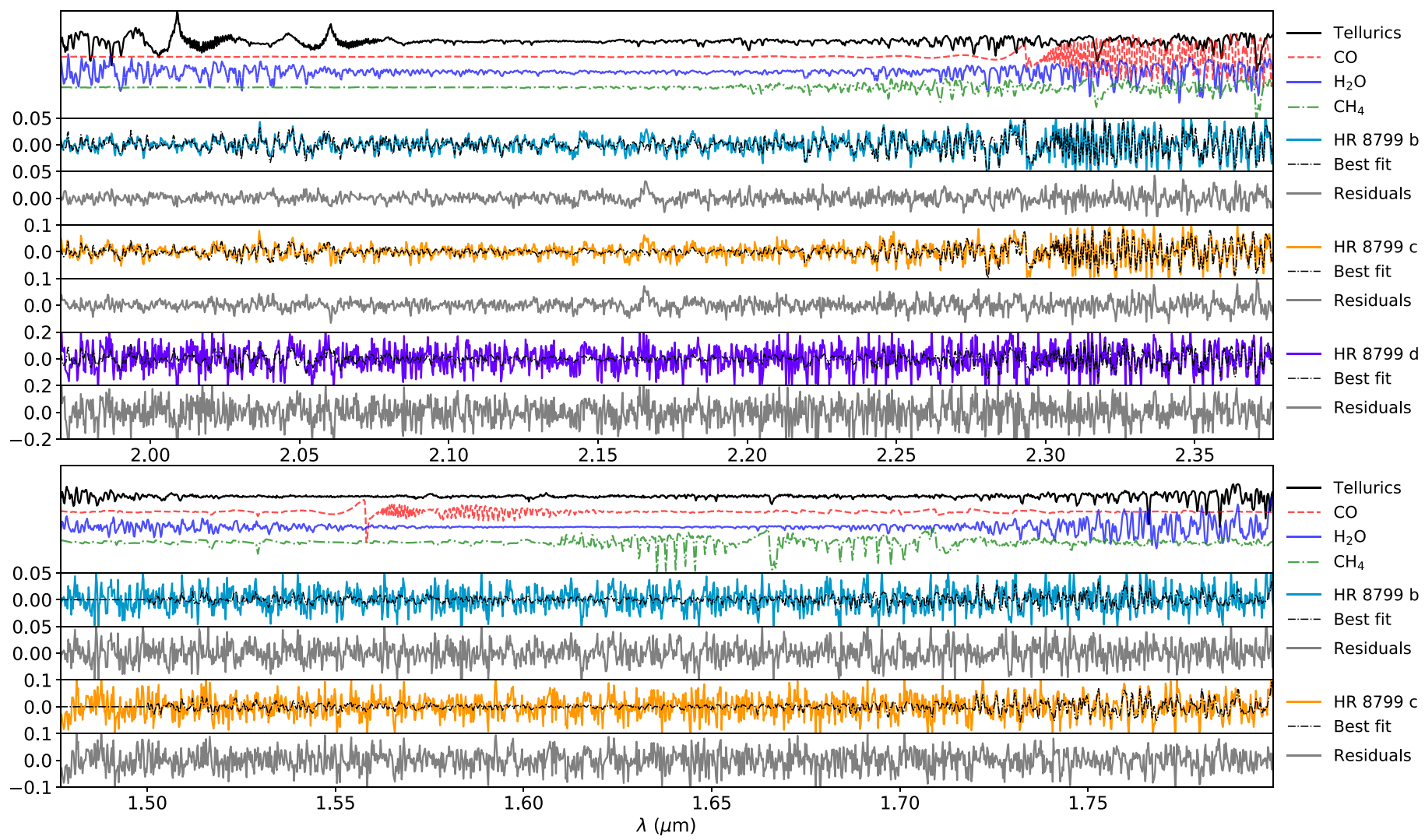

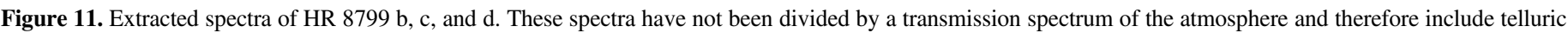

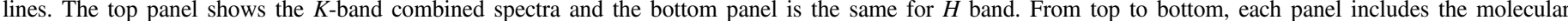

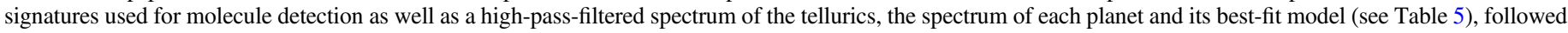

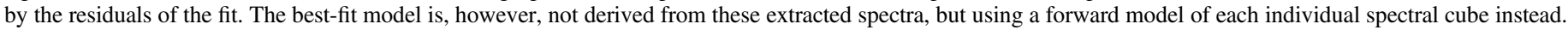

(The data used to create this figure are available.)

The downside of self-consistent models is the computational cost of generating the grid, which limits the number of parameters that can be explored. A recurring issue in atmospheric characterization of directly imaged low-mass companions is the radius or underluminosity problem (e.g., Marois et al. 2008; Bowler et al. 2010). It is not uncommon for higher effective temperatures to be favored in the models, which leads to unrealistically small planet radii due to the low luminosity of the objects. These inferred parameters are inconsistent with evolutionary models. Evidence shows that the discrepancy can be improved with better modeling of the clouds and disequilibrium chemistry (Barman et al. 2011a, 2011b; Currie et al. 2011; Skemer et al. 2011). Wang et al. (2020) also showed that extending the wavelength coverage (e.g., 3-5 $\mu \mathrm{m})$ helps to better constrain these parameters. In this work, the inferred temperatures in Table 5 from OSIRIS are higher than the evolutionary model predictions, and therefore hitting the edge of our grid at $1200 \mathrm{~K}$. Unfortunately, this systematic uncertainty cannot be self-consistently included in our estimates without additional parameters in the model (a.k.a. omitted-variable bias), which is likely a reason why formal error bars are unrealistically small. While free atmospheric retrievals are prone to overfitting, self-consistent models with few varied parameters are under fitting. Going beyond the grid approach is paramount to better model the atmosphere and include more parameters that would help resolve this issue; e.g., clouds and disequilibrium chemistry.

Another potential problem plaguing inference of atmospheric parameters is the data calibration and modeling. The high contrast between the planet and the star can result in residual starlight in the planet spectrum. To only cite a few examples, photometric calibration can be challenging, speckle noise is common place in lower resolution spectra, and the telluric lines can add systematics to the data at higher resolution. In Appendix B, we identify a moderate level of correlation between neighboring pixels in high-pass-filtered OSIRIS spectra, which could point toward improper calibrations of the wavelength solution and the resolution of the instrument or correlated noise from the interpolation in the spectral cube reconstruction from the detector images. The need for principal components in the forward model, the residual stellar Brackett- $\gamma$ in Figure 11, or the outliers in Figures 5 and 12 are all signs of imperfect modeling of the data. Hierachical Bayesian models are a great way to address these issues (Section 4.2 in Czekala et al. 2017), but the limited number of free parameters in the atmospheric grid is more likely the dominant factor to explain inconsistencies between the low- and moderate-resolution spectra in this analysis (see Figure 13).

\subsection{Carbon to Oxygen Number Ratio}

In this work, we estimated the $\mathrm{C} / \mathrm{O}$ ratio of $\mathrm{HR} 8799 \mathrm{~b}$, c, and $\mathrm{d}$ using the framework described in Section 3. Out of the four planets in the system, HR $8799 \mathrm{~d}$ is the only planet that did not have a reliable $\mathrm{C} / \mathrm{O}$ measurement in the literature.

A feature of the modeling approach used is that it does not rely on a prior starlight suppression, spectral extraction, and 

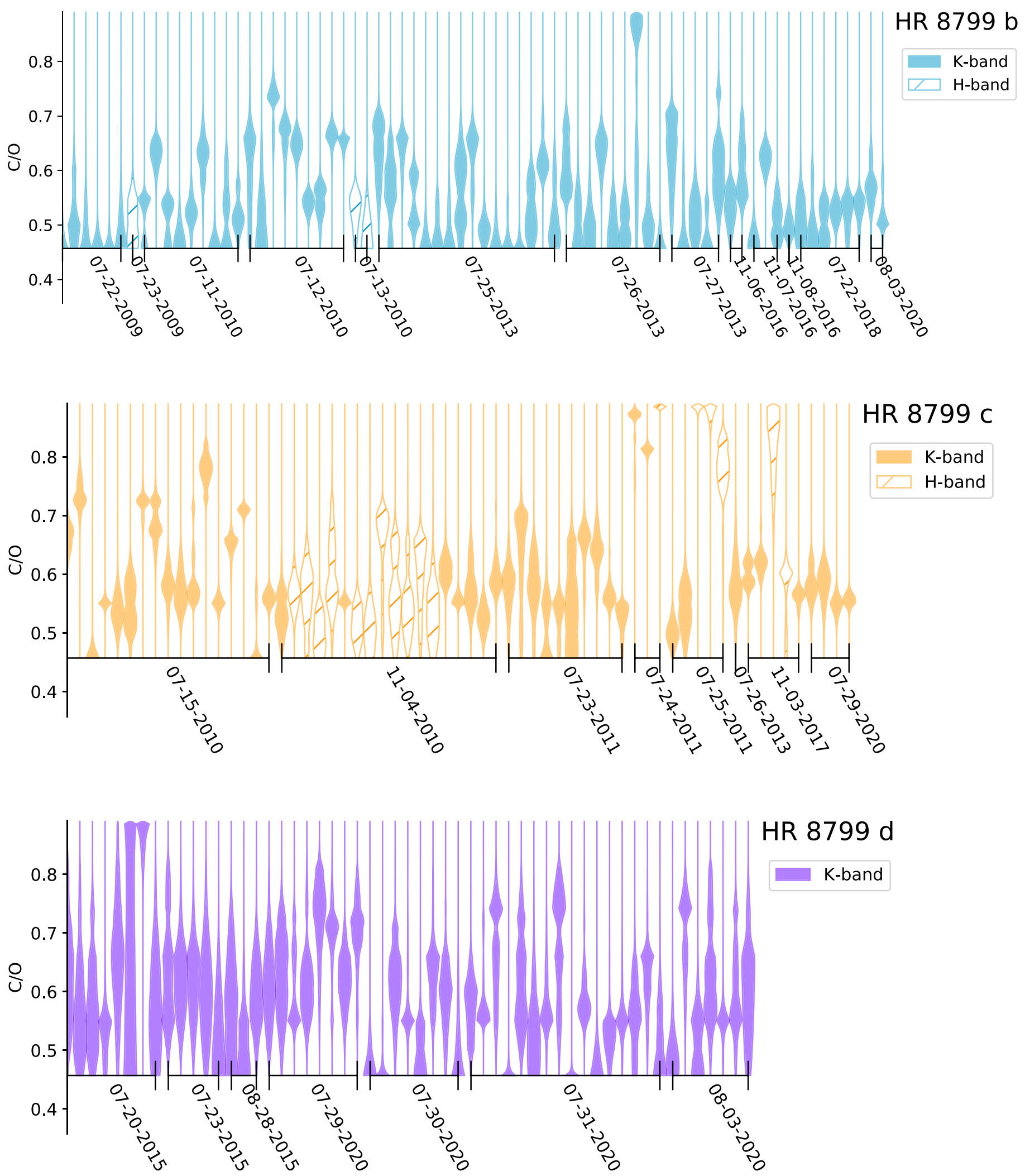

Figure 12. Marginalized C/O posteriors for each exposure of HR $8799 \mathrm{~b}$, c, and d as a function of the date of observation. The width of the hatched ( $H$-band) or shaded ( $K$-band) area is proportional to the probability density of the posterior after normalization of its peak value. The limits of the $y$-axis correspond to the edge of the atmospheric model grid along the $\mathrm{C} / \mathrm{O}$ dimension. 
......... Photometry

----. Low resolution spectra

— Forward model OSIRIS
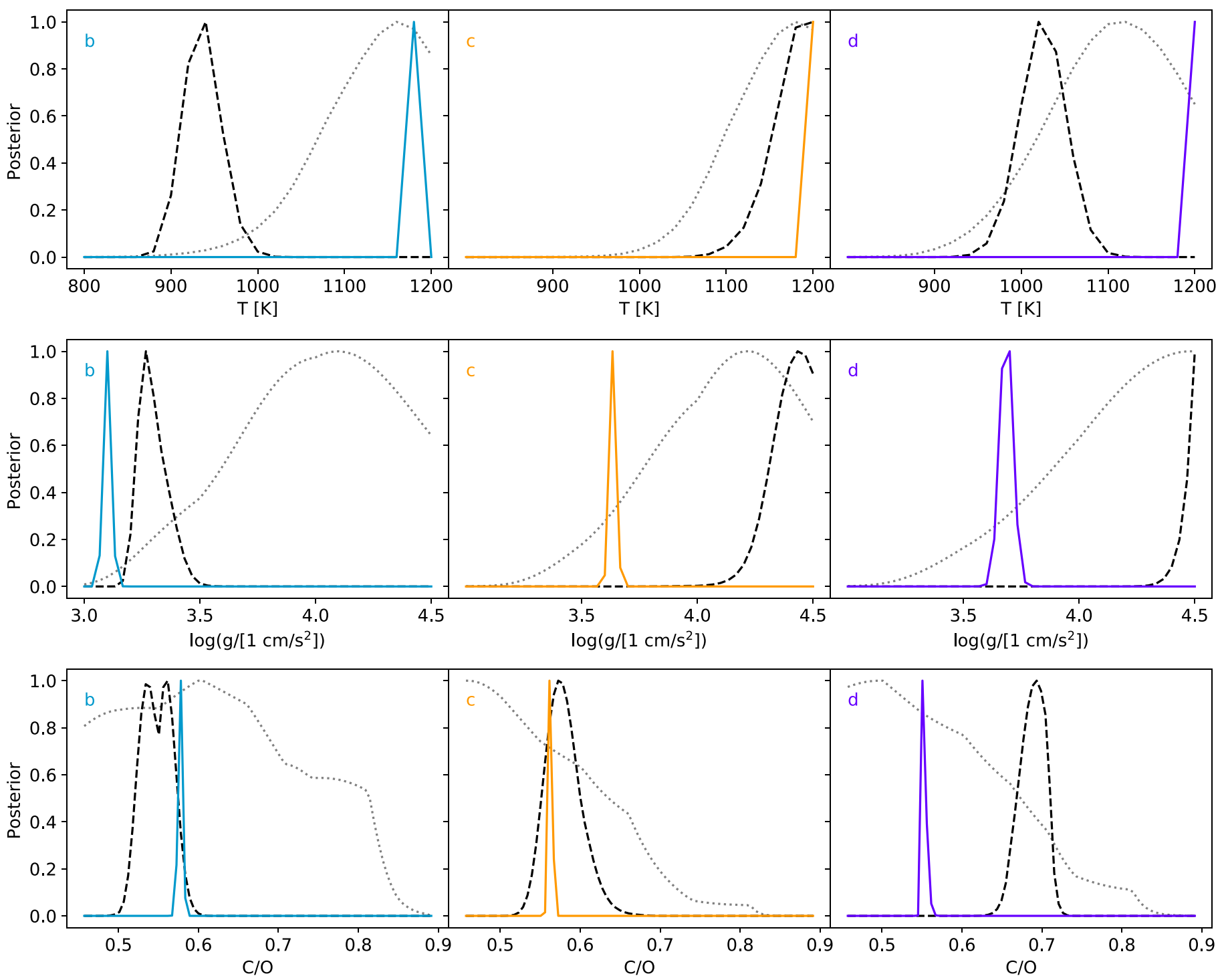

Figure 13. Marginalized posteriors for temperature, surface gravity, and C/O of HR $8799 \mathrm{~b}$, c, and d. Each row corresponds to a different parameter and each column represents to a different planet. Each panels contains three posteriors corresponding to fits to the photometry (solid line), low-resolution spectra (black and gray dashed lines), and the forward model of OSIRIS spectra (colored lines).

Table 5

Best-fit Parameters Based on the Forward Model of the OSIRIS Data Only

\begin{tabular}{llcc}
\hline \hline Planet & $T_{\text {eff }}(\mathrm{K})$ & $\log \left(g /\left[1 \mathrm{~cm} \mathrm{~s}^{-2}\right]\right)$ & $\mathrm{C} / \mathrm{O}$ \\
\hline $\mathrm{b}$ & $1180_{-14}^{+14}$ & $3.10_{-0.03}^{+0.03}$ & $0.578_{-0.005}^{+0.004}$ \\
$\mathrm{c}$ & $1200_{-14}^{*}$ & $3.63_{-0.02}^{+0.03}$ & $0.562_{-0.004}^{+0.004}$ \\
$\mathrm{~d}$ & $1200_{-14}^{*}$ & $3.7_{-0.05}^{+0.03}$ & $0.551_{-0.004}^{+0.005}$ \\
\hline
\end{tabular}

Note. The star * signifies that the best-fit value corresponds to the edge of the grid and should therefore be seen as an upper, or lower, limit instead.

telluric correction. The likelihood is directly defined from a forward model of the spectral cubes as produced by the OSIRIS data reduction pipeline. The $\mathrm{C} / \mathrm{O}$ estimates $[\mathrm{C} / \mathrm{O}]_{\mathrm{b}}=0.578_{-0.005}^{+0.008}$, $[\mathrm{C} / \mathrm{O}]_{\mathrm{c}}=0.562_{-0.004}^{+0.007}$, and $\left.[\mathrm{C} / \mathrm{O}]_{\mathrm{d}}=0.55_{-0.014}^{+0.011}\right)$ are consistent with past work from our group $(\mathrm{C} / \mathrm{O}]_{\mathrm{b}}=0.66_{-0.08}^{+0.04},[\mathrm{C} / \mathrm{O}]_{\mathrm{c}}=$ $0.562_{-0.11}^{+0.14}$; Konopacky et al. 2013; Barman et al. 2015). As discussed previously, the uncertainties are underestimated but they really emphasize the constraining power of these spectra. We note that $\mathrm{C} / \mathrm{O}$ is the most consistent of the three parameters in Figure 13 with the only discrepancy being for the GPI spectrum of HR 8799 d.

Stellar C/Os were also found by Lavie et al. (2017) for HR 8799 c $\left([\mathrm{C} / \mathrm{O}]_{\mathrm{c}}=0.55_{-0.12}^{0.1}\right)$, Wang et al. (2020) $[\mathrm{C} / \mathrm{O}]_{\mathrm{c}}=$ $0.58_{-0.06}^{0.06}$ with weak priors), and by Mollière et al. (2020) for HR 8799 e $\left([C / O]_{e}=0.6_{-0.08}^{+0.07}\right)$. As discussed in Section 8.3, such atmospheric retrievals are powerful, because they can marginalize over a wider range of parameters including clouds, which is a limitation in the grid based methodology used here. However, the computational cost of going to high spectral resolution can become prohibitive. We note the discrepancy between the OSIRIS inferred C/O of HR 8799 b and the results from atmospheric retrieval of low-resolution spectra, which are close to unity: $[\mathrm{C} / \mathrm{O}]_{\mathrm{b}}=0.92 \pm 0.01$ (Lavie et al. 2017) and 


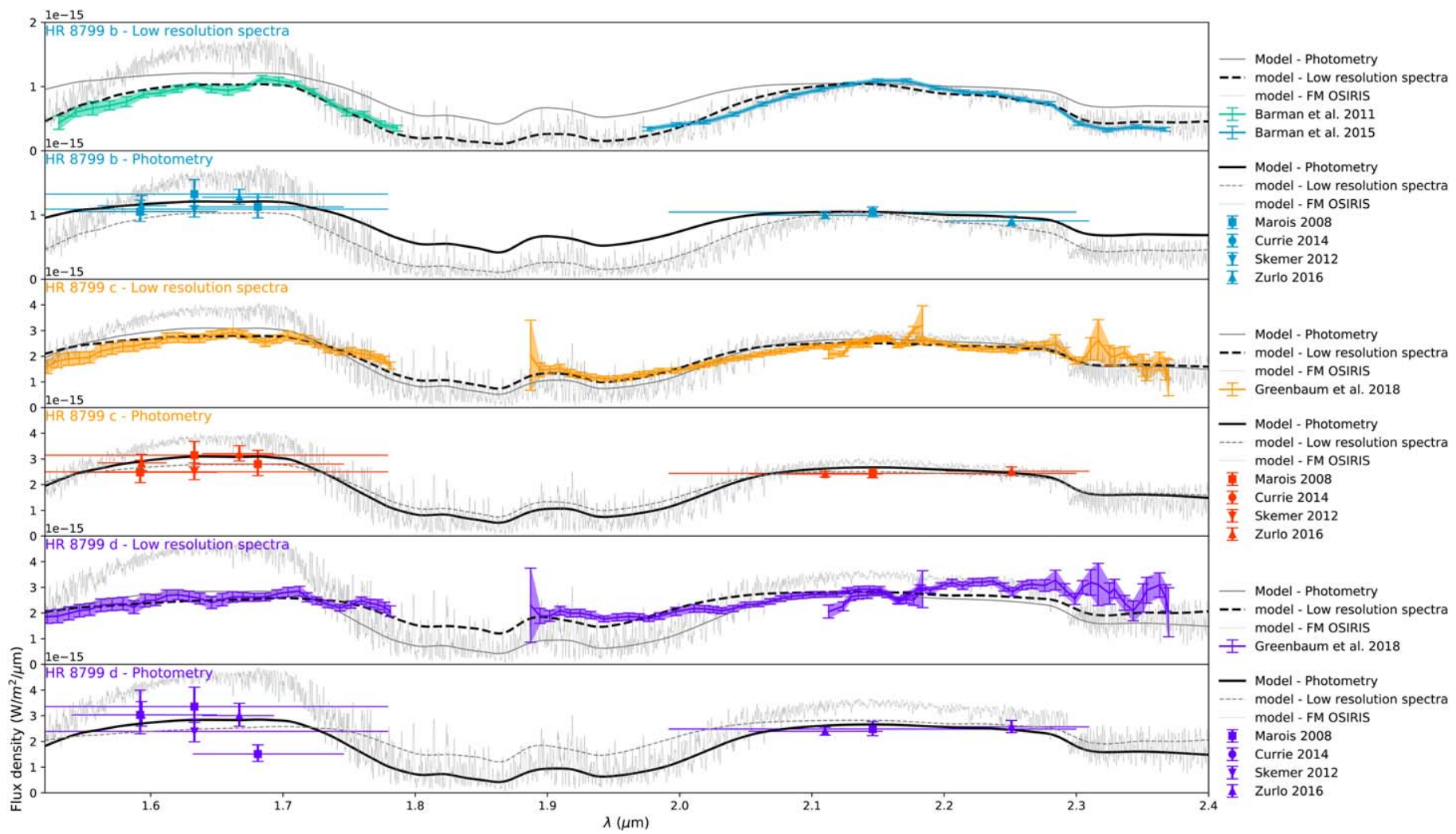

Figure 14. Published photometry and low-resolution spectra for HR 8799 b, c, and d. Each panel contains three best-fit models respectively from a fit to the photometry (solid), the low-resolution spectra (dashed), and the OSIRIS data presented in this work (dotted). The best-fit models for the photometry and the lowresolution spectra were convolved to a resolution of $R=100$ in this visualization. In each panel, the best-fit model associated to the data being plotted is drawn with a thick black line as opposed to gray. The data used in this plot are listed in Table 6.

Table 6

Published $H$ - and $K$-band Absolute Magnitudes and Low-resolution Spectra for HR 8799 b, c, and d

\begin{tabular}{|c|c|c|c|c|c|c|c|c|}
\hline \multirow{2}{*}{$\begin{array}{l}\text { Filter } \\
\text { Photometry }\end{array}$} & \multirow{2}{*}{$\lambda$} & \multicolumn{2}{|c|}{ HR 8799 b } & \multicolumn{2}{|c|}{ HR 8799 c } & \multicolumn{2}{|c|}{ HR $8799 \mathrm{~d}$} & \multirow{2}{*}{ References } \\
\hline & & Mag. & Err. & Mag. & Err. & Mag. & Err. & \\
\hline$H 2$ & 1.593 & 15.1 & 0.14 & 14.11 & 0.12 & 14.04 & 0.17 & Zurlo et al. (2016) \\
\hline$H 3$ & 1.667 & 14.8 & 0.1 & 13.8 & 0.1 & 13.87 & 0.16 & Zurlo et al. (2016) \\
\hline$K 1$ & 2.11 & 14.17 & 0.06 & 13.21 & 0.05 & 13.22 & 0.07 & Zurlo et al. (2016) \\
\hline$K 2$ & 2.251 & 13.99 & 0.09 & 12.88 & 0.07 & 12.86 & 0.1 & Zurlo et al. (2016) \\
\hline$H$ & 1.633 & 14.87 & 0.17 & 13.93 & 0.17 & 13.86 & 0.22 & Marois et al. (2008) ${ }^{a}$ \\
\hline $\mathrm{CH} 4 \mathrm{~S}$ & 1.592 & 15.18 & 0.17 & 14.25 & 0.19 & 14.03 & 0.3 & Marois et al. (2008) \\
\hline $\mathrm{CH} 4 \mathrm{~L}$ & 1.681 & 14.89 & 0.18 & 13.9 & 0.19 & 14.57 & 0.23 & Marois et al. (2008) \\
\hline Ks & 2.146 & 14.05 & 0.08 & 13.13 & 0.08 & 13.11 & 0.12 & Marois et al. (2008) \\
\hline$H$ & 1.633 & 15.08 & 0.13 & 14.18 & 0.14 & 14.23 & 0.2 & Skemer et al. (2012) ${ }^{a}$ \\
\hline Spectra & $\lambda$ & \multicolumn{2}{|c|}{$R=\lambda / \delta \lambda$} & $\mathrm{b}$ & $\mathrm{c}$ & c & \multicolumn{2}{|r|}{ Reference } \\
\hline$H$ & $1.48-1.82$ & \multicolumn{2}{|c|}{46} & & $x$ & \multirow{2}{*}{\multicolumn{2}{|c|}{$\begin{array}{l}\times \\
\times\end{array}$}} & \\
\hline$K 1$ & $1.86-2.22$ & \multicolumn{2}{|c|}{66} & & $x$ & & & GPI; Greenbaum et al. (2018) \\
\hline$K 2$ & $2.08-2.43$ & \multicolumn{2}{|c|}{79} & & $x$ & \multirow{2}{*}{\multicolumn{2}{|c|}{$x$}} & GPI; Greenbaum et al. (2018) \\
\hline $\mathrm{Hbb}$ & $1.45-1.83$ & \multirow{2}{*}{\multicolumn{2}{|c|}{200}} & \multicolumn{2}{|l|}{$x$} & & & OSIRIS; Barman et al. (2011a) \\
\hline $\mathrm{Kbb}$ & $1.94-2.42$ & & & $x$ & & & & OSIRIS; Barman et al. (2011a) \\
\hline
\end{tabular}

Note.

a The filters of these photometry poinst are broader than the definition of the model spectra. They are therefore not included in the fits but shown in Figure 14.

$[\mathrm{C} / \mathrm{O}]_{\mathrm{b}}=0.97 \pm 0.01$ (Lee et al. 2013). Such high values of the $\mathrm{C} / \mathrm{O}$ are ruled out by Barman et al. (2015) and the high S/N CO band-head measurement from OSIRIS.

Despite the possible systematics, our analysis suggests that HR 8799 d has a similar C/O to HR 8799 b and c. Combined with literature values, it can conclude that all four known planets orbiting HR 8799 have a stellar C/O ratio. We discuss the implication on planet formation in the Section 8.5.

\subsection{Implication for Planet Formation Models}

Based on our work with OSIRIS for HR 8799 b, c, and d, and the recent analysis of HR 8799e by Mollière et al. (2020), all four planets in HR 8799 have $\mathrm{C} / \mathrm{O}$ ratios consistent with the host star $\left(\mathrm{C} / \mathrm{O}=0.54_{-0.09}^{+0.12}\right.$; Wang et al. 2020). Though systematic uncertainties remain in the models which might alter these values somewhat, all analysis and extracted spectra point to spectral 
morphologies that do not vary in any significant way from solar values. We note that Mollière et al. (2020) performed a retrieval analysis in which they also derived an enhanced bulk metallicity relative for $\mathrm{HR} 8799$ e $([\mathrm{Fe} / \mathrm{H}] \sim 0.5)$. We have not fit for metallicity, but solar metallicity templates appear to be a fine match to all of our spectra. The CO lines, for instance, do not appear to be significantly deeper than a solar metallicity, stellar $\mathrm{C} / \mathrm{O}$ ratio requires. However, because $H$ - and $K$-band spectra are primarily sensitive to the abundance of carbon and oxygen, future observations of shorter wavelengths, such as $J$ band, where additional species such as potassium and iron (via $\mathrm{FeH}$ ) may be present could shed light on the bulk metallicity of the planets.

The measurement of a consistent $\mathrm{C} / \mathrm{O}$ ratio for all four planets is an important milestone and result. For all practical purposes, these values do not sufficiently deviate from stellar $\mathrm{C} / \mathrm{O}$ to be decisive with respect to specific formation scenarios. A stellar ratio is consistent both with formation through gravitational instability, or with core/pebble accretion scenarios that include planetesimal enrichment. Indeed, it is clear that when taking into account the impact of a few of the many parameters that might impact this ratio in a planetary atmosphere, including pebble drift across ice lines and planet migration, almost any value of $\mathrm{C} / \mathrm{O}$ (and $\mathrm{C} / \mathrm{H}$ and $\mathrm{O} / \mathrm{H}$ ) is plausible (e.g., Mordasini et al. 2016; Booth et al. 2017; Cridland et al. 2017; Madhusudhan et al. 2017). Our goal is to begin to compile a statistical ensemble of $\mathrm{C} / \mathrm{O}$ ratio measurements for the directly imaged planets in order to begin to constrain which core/pebble scenarios may be feasible, starting with the four HR 8799 planets. If it is the case that gas giants that form via core/pebble accretion should notionally have an elevated $\mathrm{C} / \mathrm{O}$ ratio in their atmospheres after runaway gas accretion, a clear possibility here is that these very large gas giant planets like the HR 8799 planets (see also $\kappa$ Andromedae b, Wilcomb et al. 2020) must also undergo extremely significant planetesimal enrichment in order to grow so large, leading to a stellar $\mathrm{C} / \mathrm{O}$.

Mollière et al. (2020) describe a formation scenario in which all of the HR 8799 planets form beyond the $\mathrm{CO}$ ice line as a way to achieve the observed $\mathrm{C} / \mathrm{O}$ ratios. This is predicated on an understanding of where the ice lines would have been around HR 8799. Since HR 8799 is a hotter A-type star, values based on the Sun are likely inappropriate. However, the values we assumed for the snow lines based on Öberg et al. (2011) predictions for the A0 star AB Auriga (Figure 2), which places the CO frost line $>300$ $\mathrm{au}$, is also likely incorrect for HR 8799 . The best way to estimate these locations it to use measurements of real ice lines from similar, very young stars. For instance, Qi et al. (2015) directly measure the CO snow line at 90 au around the A2 star HD 163296. HR 8799 is cooler than this star, so the CO frost line is likely interior to this. However, Qi et al. (2015) also note that the CO freezes out at a higher temperature in this disk than in TW Hydra, perhaps due to compositional differences, and suggest that actually measuring snow lines is the best way to get a true statistical handle on their location. Based on the available information, a safe assumption is that $\mathrm{CO}$ snow line in the planet-forming disk around HR 8799 was probably in the range of $\sim 50-100$ au.

If the snow line is closer to $50 \mathrm{au}$, it is certainly plausible that all four planets started their initial formation beyond that range. Indeed, the simulation shown in Johansen \& Lambrechts (2017) for the formation of HR 8799 based on pebble accretion has the cores forming between $\sim 50$ and $160 \mathrm{au}$. However, in this simulation the bulk to the runaway accretion of the envelope for both HR 8799 e and HR 8799 d occurs interior to 50 au as the planets all migrate inward to their current location. The fact that the current planet locations span more than one frost line complicates the overall picture of the formation history of the HR 8799 system.

Altogether, the results presented here and also in Wilcomb et al. (2020) have not yet offered direct evidence of core/pebble accretion formation for directly imaged planets, which would have come in the form of an elevated $\mathrm{C} / \mathrm{O}$ ratio. While we still seek to measure this ratio for all directly imaged planets currently discovered, if all values end up being consistent with stellar, additional formation diagnostics will be needed to shed more light on the formation of wide gas giants. Bulk metallicity and nitrogen abundance, as suggested by Mollière et al. (2020) based on Öberg \& Wordsworth (2019) and Bosman et al. (2019) as well as Turrini et al. (2021), are two possible diagnostics to pursue next.

\section{Summary and Conclusion}

We performed a comprehensive uniform reanalysis of the HR 8799 data acquired by Keck/OSIRIS, a $R \approx 4000$ infrared integral field spectrograph, at $H(1.97-2.38 \mu \mathrm{m})$ and $K$ band $(1.47-1.80 \mu \mathrm{m})$. The highlights of the this work are the detection and deep observations of HR $8799 \mathrm{~d}$, the secondclosest known planet in the system, as well as the new $H$-band data for HR 8799 c.

Using our recently developed framework (Ruffio 2019; Ruffio et al. 2019; Wilcomb et al. 2020), we first looked for signatures of water $\left(\mathrm{H}_{2} \mathrm{O}\right)$, carbon monoxide $(\mathrm{CO})$, and methane $\left(\mathrm{CH}_{4}\right)$ in the atmosphere of HR $8799 \mathrm{~b}$, c, and d. $\mathrm{H}_{2} \mathrm{O}$ and $\mathrm{CO}$ were unambiguously detected in the three cases at high $\mathrm{S} / \mathrm{N}$. Due to conflicting results, we remain unable to unambiguously conclude on the detection of methane in the atmosphere of HR $8799 \mathrm{~b}$. We note that a lower abundance of methane would suggest a more efficient vertical mixing with an eddy diffusion coefficient $\log \left(K_{\mathrm{zz}}\right) \geqslant 7$.

Then, we measured the radial velocity of HR $8799 \mathrm{~d}$ and revisited the estimates of HR $8799 \mathrm{~b}$ and c from Ruffio et al. (2019) using an improved data model to better correct for systematics. The RV of the three planets are consistent with predictions from astrometric monitoring of the system as well as coplanar and stable orbits (Wang et al. 2018b). We also showed how the planetary RVs allow better constraints on the orientation of the orbital plane of the system and the eccentricity of HR 8799 b, c, and d.

Finally, we fit a grid of atmosphere model with varying effective temperature, surface gravity, and $\mathrm{C} / \mathrm{O}$. Unfortunately, such measurements are still dominated by modeling uncertainties that are difficult to estimate, but they rule out significant deviations from stellar $\mathrm{C} / \mathrm{O}$ for the three planets. A stellar $\mathrm{C} / \mathrm{O}$ does not point to a specific formation scenario as both gravitational instability or core/pebble accretion are consistent with such value. However, if core accretion is the mechanism by which these planets formed, then a significant planetesimal enrichment is likely.

We would like to thank Karin Öberg for her helpful discussions about planet formation. J.-B.R. acknowledges support from the David and Ellen Lee Prize Postdoctoral Fellowship. The research was supported by grants from NSF, including AST-1411868 (J.-B.R., B.M.) and 1614492 (T.S.B.). Material presented in this work is supported by the National Aeronautics and Space Administration under Grants/Contracts/Agreements No. NNX17AB63G (Q.M.K., T.S.B., and K.K.W.) issued through the Astrophysics Division of the Science Mission Directorate and NNX15AD95G (J.-B.R., R.J.D.R.). Any opinions, findings, and conclusions or recommendations expressed in this work are those 
of the author(s) and do not necessarily reflect the views of the National Aeronautics and Space Administration. This work benefited from NASA's Nexus for Exoplanet System Science (NExSS) research coordination network sponsored by NASA's Science Mission Directorate. This work made use of the sky background models made available by the Gemini observatory ${ }^{17}$. This research has made use of the SVO Filter Profile Service (http://svo2.cab.inta-csic.es/theory/fps/) supported from the Spanish MINECO through grant AYA2017-84089. The W. M. Keck Observatory is operated as a scientific partnership among the California Institute of Technology, the University of California, and NASA. The Keck Observatory was made possible by the generous financial support of the W. M. Keck Foundation. We also wish to recognize the very important cultural role and reverence that the summit of Maunakea has always had within the indigenous Hawaiian community. We are most fortunate to have the opportunity to conduct observations from this mountain.

Facility: Keck I (OSIRIS). Software: astropy ${ }^{18}$ (Astropy Collaboration et al. 2013), Matplotlib ${ }^{19}$ (Hunter 2007), orbitize! ${ }^{20}$ (Blunt et al. 2019), ptemcee ${ }^{21}$ (Vousden et al. 2016; Foreman-Mackey et al. 2013), whereistheplanet ${ }^{22}$ (Wang et al. 2021).

\section{Appendix A Observations Summary Table}

The observing nights summary can be found in Table 7 for the science and in Tables 8 for the telluric standards. Figure 15 show S/N maps of HR $8799 \mathrm{~d}$ at the expected RV of the star for each exposure. These detection maps are computed according to Section 3.6.

Table 7

Night-by-night Summary of the HR 8799 Observations with OSIRIS/Keck

\begin{tabular}{|c|c|c|c|c|c|c|c|c|}
\hline Planet & Date & Band & Cubes & $\begin{array}{c}\text { Time } \\
(\mathrm{hr})\end{array}$ & Skies & $\begin{array}{c}\text { RV } \\
\text { Mean } \\
\text { Offset } \\
\left(\mathrm{km} \mathrm{s}^{-1}\right)\end{array}$ & $\begin{array}{c}\text { RV } \\
\text { Residual } \\
\text { Error } \\
\left(\mathrm{km} \mathrm{s}^{-1}\right)\end{array}$ & Notes \\
\hline \multirow[t]{15}{*}{$\mathrm{b}$} & 2009-07-22 & $\mathrm{Kbb}$ & 12 & 2.8 & 1 & 3.1 & 4 & Not used for RV \\
\hline & $2009-07-23$ & $\mathrm{Hbb}$ & 6 & 1.5 & 1 & 3.3 & 4 & Not used for RV \\
\hline & 2009-07-30 & $\mathrm{Hbb}$ & 8 & 2.0 & 3 & 4.9 & 4 & $\begin{array}{l}\text { No detection. } \\
\text { Cooling issue. }\end{array}$ \\
\hline & 2009-09-03 & $\mathrm{Hbb}$ & 12 & 2.8 & 1 & -5.3 & 4 & No detection. \\
\hline & 2010-07-11 & $\mathrm{Kbb}$ & 9 & 2.2 & 2 & 6.5 & 2.2 & \\
\hline & 2010-07-12 & $\mathrm{Kbb}$ & 9 & 2.2 & 1 & 5.3 & 2.2 & \\
\hline & $2010-07-13$ & $\mathrm{Hbb}$ & 2 & 0.5 & 1 & 2.9 & 1.1 & \\
\hline & 2013-07-25 & $\mathrm{Kbb}$ & 16 & 2.7 & 2 & 2.7 & 1.6 & \\
\hline & $2013-07-26$ & $\mathrm{Kbb}$ & 9 & 1.5 & 3 & 3.4 & 1.6 & \\
\hline & 2013-07-27 & $\mathrm{Kbb}$ & 7 & 1.2 & 2 & 3.1 & 1.6 & \\
\hline & 2016-11-06 & $\mathrm{Kbb}$ & 2 & 0.3 & 2 & 6.9 & 0.6 & \\
\hline & 2016-11-07 & $\mathrm{Kbb}$ & 3 & 0.5 & 2 & 6.9 & 0.6 & \\
\hline & 2016-11-08 & $\mathrm{Kbb}$ & 1 & 0.2 & 1 & 6.9 & 0.6 & \\
\hline & $2018-07-22$ & $\mathrm{Kbb}$ & 6 & 0.5 & 1 & -0.8 & 1.0 & 35 mas platescale \\
\hline & 2020-08-03 & $\mathrm{Kbb}$ & 2 & 0.3 & 3 & 27.9 & 2 & \\
\hline \multirow[t]{13}{*}{$\mathrm{c}$} & $2010-07-15$ & Kbb & 17 & 2.8 & 3 & 5.7 & 2.2 & \\
\hline & $2010-10-28$ & $\mathrm{Hbb}$ & 5 & 0.8 & 1 & 2.0 & 1.1 & No detection. \\
\hline & 2010-11-04 & $\mathrm{Hbb}$ & 12 & 2.0 & 2 & 2.9 & 1.1 & \\
\hline & & $\mathrm{Kbb}$ & 8 & 1.3 & 2 & 5.7 & 2.2 & \\
\hline & 2011-07-23 & $\mathrm{Kbb}$ & 12 & 2.0 & 3 & 3.4 & 2.9 & \\
\hline & 2011-07-24 & $\mathrm{Hbb}$ & 12 & 2.0 & 3 & 0.8 & 1.5 & \\
\hline & & $\mathrm{Kbb}$ & 3 & 0.5 & 1 & 3.4 & 2.9 & \\
\hline & 2011-07-25 & $\mathrm{Hbb}$ & 14 & 2.3 & 3 & 0.8 & 1.5 & \\
\hline & & $\mathrm{Kbb}$ & 2 & 0.3 & 2 & 3.4 & 2.9 & \\
\hline & 2013-07-26 & $\mathrm{Kbb}$ & 1 & 0.2 & 3 & 3.4 & 1.6 & \\
\hline & 2017-11-03 & $\mathrm{Hbb}$ & 14 & 2.3 & 3 & -11.4 & 0.8 & \\
\hline & & $\mathrm{Kbb}$ & 3 & 0.5 & 2 & -13.4 & 1.0 & \\
\hline & 2020-07-29 & $\mathrm{Kbb}$ & 4 & 0.7 & 3 & 27.1 & 2 & \\
\hline \multirow[t]{9}{*}{$\mathrm{d}$} & 2013-07-27 & $\mathrm{Kbb}$ & 1 & 0.2 & 2 & 3.1 & 1.6 & No detection. \\
\hline & $2015-07-20$ & $\mathrm{Kbb}$ & 15 & 2.5 & 4 & -2.7 & 1.6 & \\
\hline & $2015-07-22$ & $\mathrm{Kbb}$ & 15 & 2.5 & 4 & -2.3 & 1.6 & No detection. \\
\hline & $2015-07-23$ & $\mathrm{Kbb}$ & 15 & 2.5 & 4 & -2.7 & 1.6 & \\
\hline & $2015-08-28$ & $\mathrm{Kbb}$ & 18 & 3.0 & 2 & -1.9 & 1.6 & \\
\hline & 2020-07-29 & $\mathrm{Kbb}$ & 10 & 1.7 & 3 & 27.1 & 2 & \\
\hline & $2020-07-30$ & $\mathrm{Kbb}$ & 13 & 2.2 & 3 & 26.7 & 2 & \\
\hline & $2020-07-31$ & $\mathrm{Kbb}$ & 18 & 3.0 & 3 & 26.7 & 2 & \\
\hline & $2020-08-03$ & $\mathrm{Kbb}$ & 15 & 2.5 & 3 & 27.9 & 2 & \\
\hline
\end{tabular}

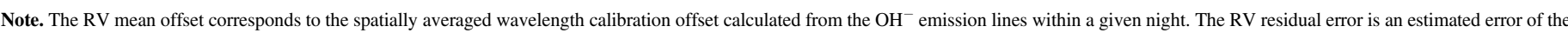
spatially dependent wavelength offset relative to the spatial average (see Ruffio et al. 2019).

\footnotetext{
$\overline{17}$ http://www.gemini.edu/sciops/telescopes-and-sites/observing-conditionconstraints/ir-background-spectra
}

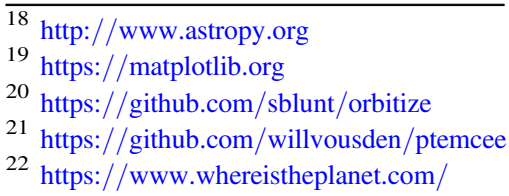


Table 8

List of Standard Star Observations Used in This Work

\begin{tabular}{|c|c|c|c|c|c|}
\hline Date & Star Name & Band & Cubes & $\begin{array}{l}\text { Int. Time } \\
\quad(\mathrm{s})\end{array}$ & Mode \\
\hline \multirow[t]{2}{*}{$2009-07-22$} & BD+14 4774 & $\mathrm{Kbb}$ & 3 & 30.0 & PSF \\
\hline & HD 210501 & Kbb & 3 & 30.0 & flux only \\
\hline \multirow[t]{2}{*}{$2009-07-23$} & BD+14 4774 & $\mathrm{Hbb}$ & 4 & 30.0 & PSF \\
\hline & HD 210501 & $\mathrm{Hbb}$ & 6 & 30.0 & flux only \\
\hline \multirow[t]{2}{*}{ 2009-07-30 } & BD+14 4774 & $\mathrm{Hbb}$ & 1 & 30.0 & PSF \\
\hline & $\mathrm{BD}+144774$ & $\mathrm{Hbb}$ & 2 & 30.0 & flux only \\
\hline \multirow[t]{2}{*}{ 2009-09-03 } & BD+14 4774 & $\mathrm{Hbb}$ & 3 & 30.0 & PSF \\
\hline & HD 7215 & $\mathrm{Hbb}$ & 3 & 3.0 & PSF \\
\hline \multirow[t]{3}{*}{ 2010-07-11 } & HD 210501 & $\mathrm{Kbb}$ & 5 & 10.0 & PSF \\
\hline & HIP 1123 & Kbb & 3 & 2.0 & PSF \\
\hline & HR 8799 & $\mathrm{Kbb}$ & 2 & 2.0 & PSF \\
\hline \multirow[t]{2}{*}{ 2010-07-12 } & HD 210501 & Kbb & 3 & 30.0 & PSF \\
\hline & HIP 1123 & Kbb & 3 & 2.0 & PSF \\
\hline \multirow[t]{2}{*}{ 2010-07-13 } & HIP 1123 & $\mathrm{Hbb}$ & 3 & 2.0 & PSF \\
\hline & HR 8799 & $\mathrm{Hbb}$ & 2 & 2.0 & PSF \\
\hline \multirow[t]{2}{*}{ 2010-07-15 } & HD 210501 & Kbb & 2 & 30.0 & PSF \\
\hline & HIP 1123 & $\mathrm{Kbb}$ & 3 & 2.0 & PSF \\
\hline $2010-10-28$ & HD 210501 & $\mathrm{Hbb}$ & 3 & 30.0 & PSF \\
\hline \multirow[t]{5}{*}{ 2010-11-04 } & HD 210501 & $\mathrm{Hbb}$ & 4 & 30.0 & PSF \\
\hline & HIP 1123 & $\mathrm{Hbb}$ & 3 & 2.0 & PSF \\
\hline & HIP 116886 & $\mathrm{Hbb}$ & 3 & 30.0 & PSF \\
\hline & HD 210501 & $\mathrm{Kbb}$ & 6 & 30.0 & PSF \\
\hline & HIP 1123 & Kbb & 3 & 2.0 & PSF \\
\hline \multirow[t]{2}{*}{ 2011-07-23 } & HD 210501 & Kbb & 3 & 30.0 & PSF \\
\hline & HIP 1123 & Kbb & 3 & 2.0 & PSF \\
\hline \multirow[t]{3}{*}{ 2011-07-24 } & HD 210501 & $\mathrm{Hbb}$ & 2 & 15.0 & PSF \\
\hline & HIP 1123 & $\mathrm{Hbb}$ & 2 & 2.0 & PSF \\
\hline & HIP 1123 & Kbb & 2 & 2.0 & PSF \\
\hline \multirow[t]{4}{*}{$2011-07-25$} & HD 210501 & $\mathrm{Hbb}$ & 3 & 5.0 & PSF \\
\hline & HIP 1123 & $\mathrm{Hbb}$ & 3 & 2.0 & PSF \\
\hline & HD 210501 & Kbb & 3 & 5.0 & PSF \\
\hline & HIP 1123 & Kbb & 3 & 2.0 & PSF \\
\hline \multirow[t]{3}{*}{$2013-07-25$} & HD 210501 & Kbb & 5 & 10.0 & PSF \\
\hline & HD 210501 & $\mathrm{Kbb}$ & 1 & 10.0 & flux only \\
\hline & HR 8799 & Kbb & 3 & 2.0 & flux only \\
\hline \multirow[t]{2}{*}{ 2013-07-26 } & HD 210501 & $\mathrm{Kbb}$ & 4 & 10.0 & PSF \\
\hline & HR 8799 & Kbb & 4 & 10.0 & flux only \\
\hline \multirow[t]{3}{*}{ 2013-07-27 } & HD 210501 & $\mathrm{Kbb}$ & 4 & 10.0 & PSF \\
\hline & HR 8799 & Kbb & 3 & 10.0 & PSF \\
\hline & HR 8799 & Kbb & 3 & 10.0 & flux only \\
\hline $2015-07-20$ & HD 210501 & Kbb & 6 & 5.0 & PSF \\
\hline $2015-07-22$ & HD 210501 & Kbb & 9 & 5.0 & PSF \\
\hline $2015-07-23$ & HD 210501 & Kbb & 6 & 5.0 & PSF \\
\hline \multirow[t]{2}{*}{ 2015-08-28 } & HD 210501 & Kbb & 4 & 5.0 & PSF \\
\hline & HR 8799 & Kbb & 3 & 2.0 & flux only \\
\hline 2016-11-06 & HD 210501 & $\mathrm{Kbb}$ & 4 & 2.0 & PSF \\
\hline \multirow[t]{2}{*}{ 2016-11-07 } & HD 210501 & Kbb & 3 & 2.0 & PSF \\
\hline & HD 210501 & $\mathrm{Kbb}$ & 1 & 2.0 & flux only \\
\hline 2016-11-08 & HD 210501 & Kbb & 3 & 2.0 & PSF \\
\hline 2017-11-03 & HD 210501 & $\mathrm{Hbb}$ & 1 & 2.0 & PSF \\
\hline & HD 210501 & $\mathrm{Hbb}$ & 6 & 2.0 & flux only \\
\hline & HD 210501 & Kbb & 4 & 2.0 & PSF \\
\hline $2018-07-22$ & HD 210501 & Kbb & 3 & 2.0 & PSF \\
\hline $2020-07-29$ & HD 210501 & $\mathrm{Kbb}$ & 5 & 2.0 & PSF \\
\hline & HR 8799 & Kbb & 8 & 2.0 & PSF \\
\hline $2020-07-30$ & HR 8799 & Kbb & 9 & 2.0 & PSF \\
\hline $2020-07-31$ & HR 8799 & $\mathrm{Kbb}$ & 15 & 2.0 & PSF \\
\hline 2020-08-03 & HR 8799 & Kbb & 18 & 2.0 & PSF \\
\hline
\end{tabular}

Note. The quoted integration time is the one of a single exposure. The last column indicates if the data were used to calibrate the PSF and the transmission (PSF) or solely the transmission (flux only). 

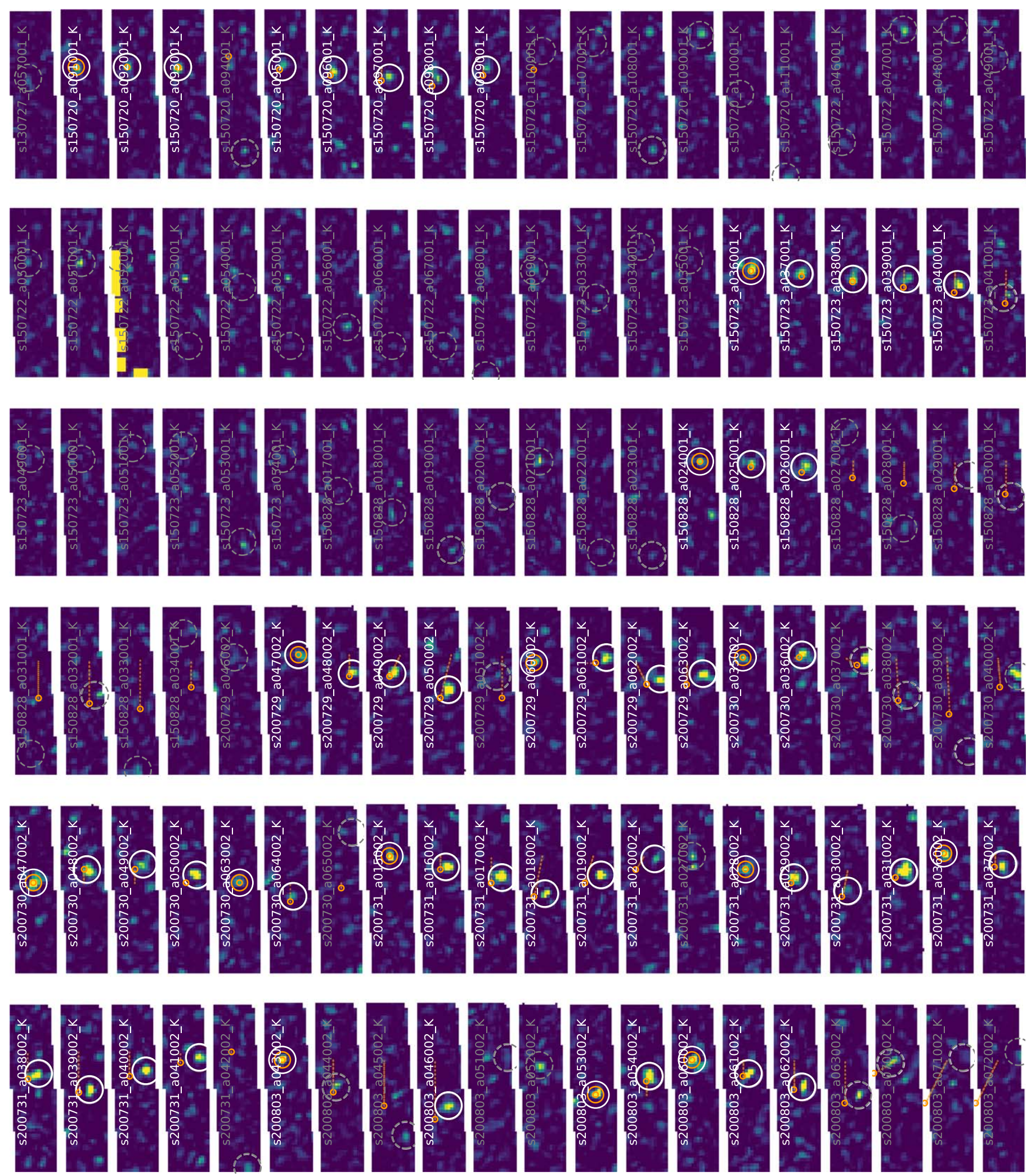

Figure 15. HR $8799 \mathrm{~d} \mathrm{~S} / \mathrm{N}$ maps for each 10 minutes exposures. The data model only includes the companion and the starlight and 10 principal components. Detections are circled in white. The gray dark circles highlight the brightest pixel in the image when there are no detections. The orange marker indicates the expected location of the planet based on the first detection in the sequence and the offsets that are saved in the fits file headers by the instrument. The offsets have a precision of only a few pixels, but they can still be used as a visual guide. 


\section{Appendix B \\ Noise Analysis}

In this appendix, we explore the noise budget for OSIRIS and our forward model at the location of HR $8799 \mathrm{~d}$. We use a single exposure from 2020 July 31 (s200731_a016002.fits) with a good detection of HR $8799 \mathrm{~d}$. We recognize the approximate nature of this analysis, and only aim at identifying the dominant source of noise.

Figure 16 illustrates the starlight suppression and noise sources for the spaxel at the location of the planet. In this particular example, the forward model is able to detect a planet that is $\sim 8$ times fainter than the starlight at the same location in only 10 minutes. We provide the OSIRIS PSF profile from the same night in Figure 17. The bottom panel of Figure 16 shows that the forward model is a good fit of the data with a standard deviation of the residuals $\sigma_{\text {res }}=0.06 \mathrm{DN}$, and that the

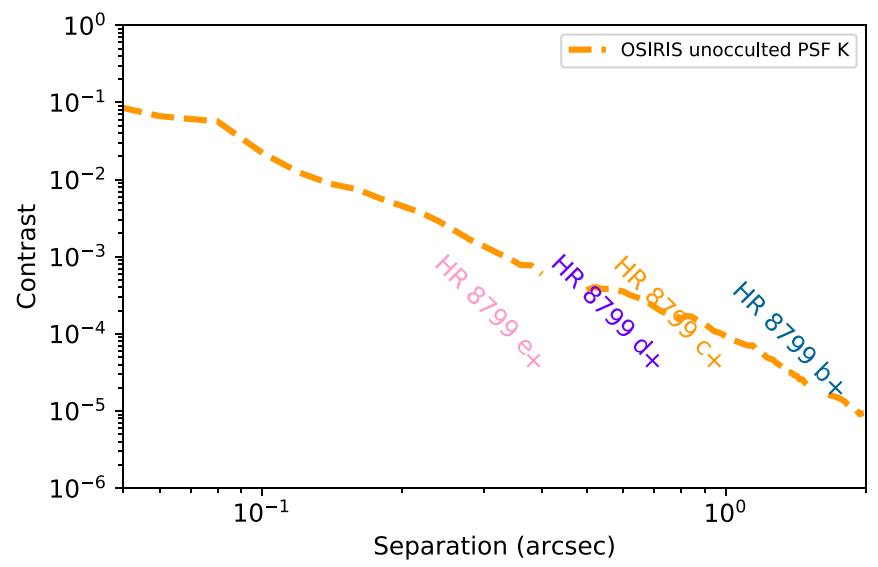

Figure 17. OSIRIS point-spread function profile compared to contrast of the HR 8799 planets.

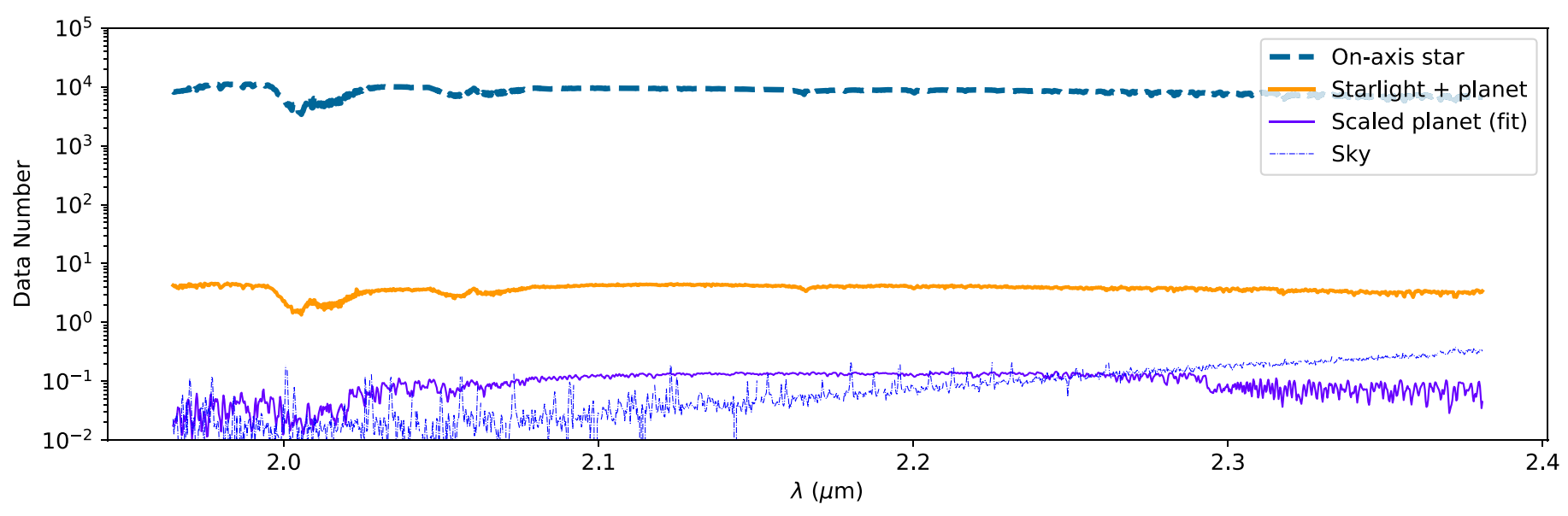

(a) Flux levels

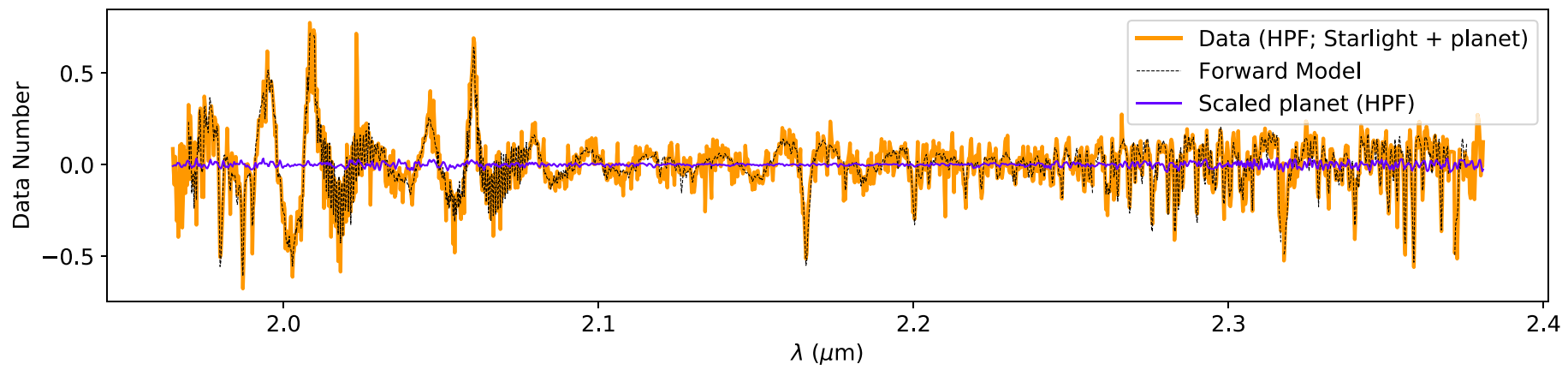

(b) Forward Modeling

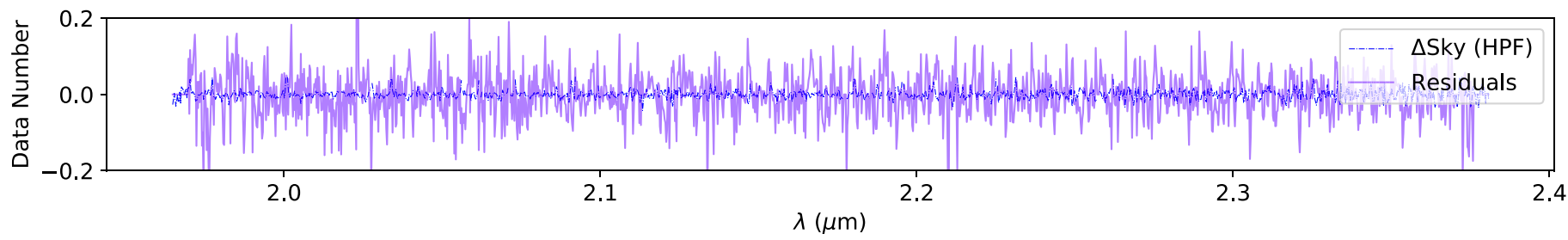

(c) Forward Modeling - Residuals

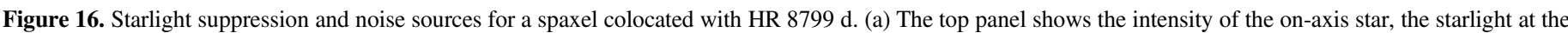

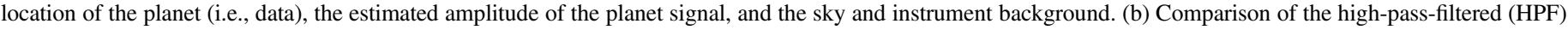

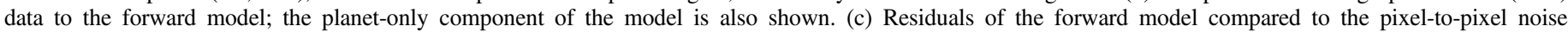

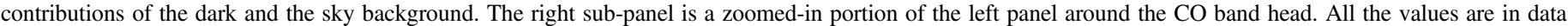
number per spaxel in a 10-minute OSIRIS spectral data cube as generated by the OSIRIS data reduction pipeline (Krabbe et al. 2004; Lyke et al. 2017). 

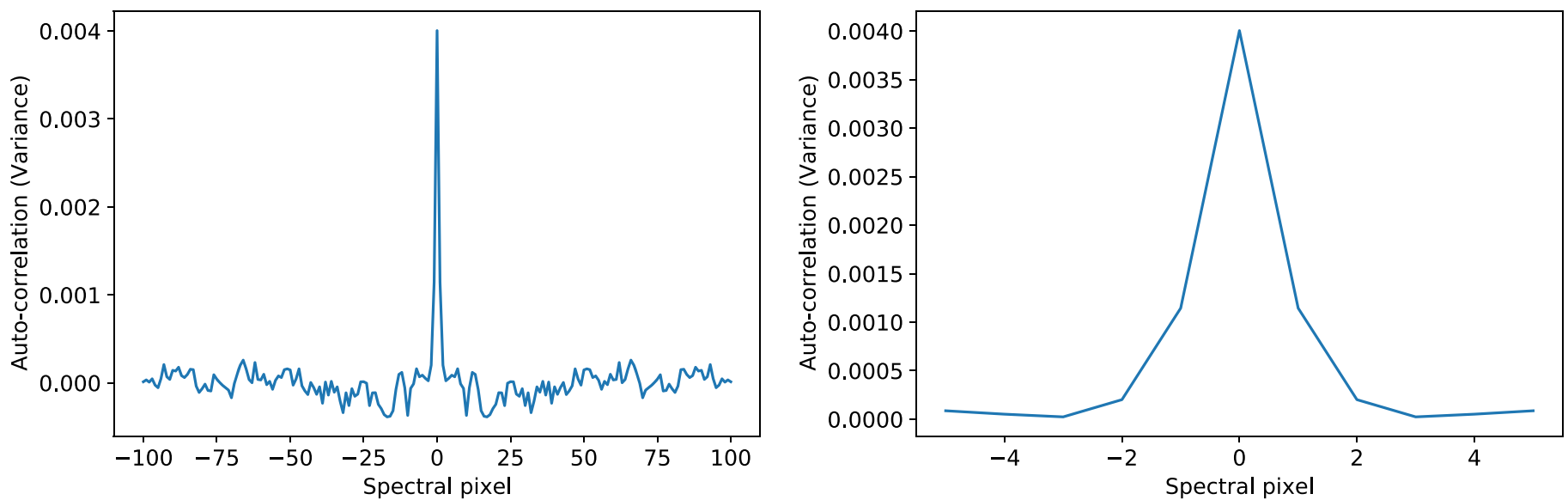

Figure 18. Autocorrelation of the residuals shown in Figure 16(d). The right panel is a close up of the central peak.

background noise $\left(\sigma_{\mathrm{bg}}=0.01 \mathrm{DN}\right)$ is negligible in this case. The background noise is estimated by taking the high-passfiltered difference of two sky cubes. The spectral bin $\mathrm{S} / \mathrm{N}$ for the planet continuum component of the signal is $\sim 2$. This $\mathrm{S} / \mathrm{N}$ is calculated by dividing the median value of the scaled planet model by the standard deviation of the residuals. The similar
$\mathrm{S} / \mathrm{N}$ for the starlight at the location of the planet is $\sim 60$. The autocorrelation of the residuals plotted in Figure 18 suggests that there is a significant correlation between neighboring pixels, which could be explained by the inteprolations in the OSIRIS data reduction pipeline. The level of the residuals is consistent with being photon noise limited. 


\section{Appendix C \\ Corner Plots}

Figure 19 include the corner plots for HR 8799 b, c, and d resulting from Section 7 and marginalized over the RV of the planet.

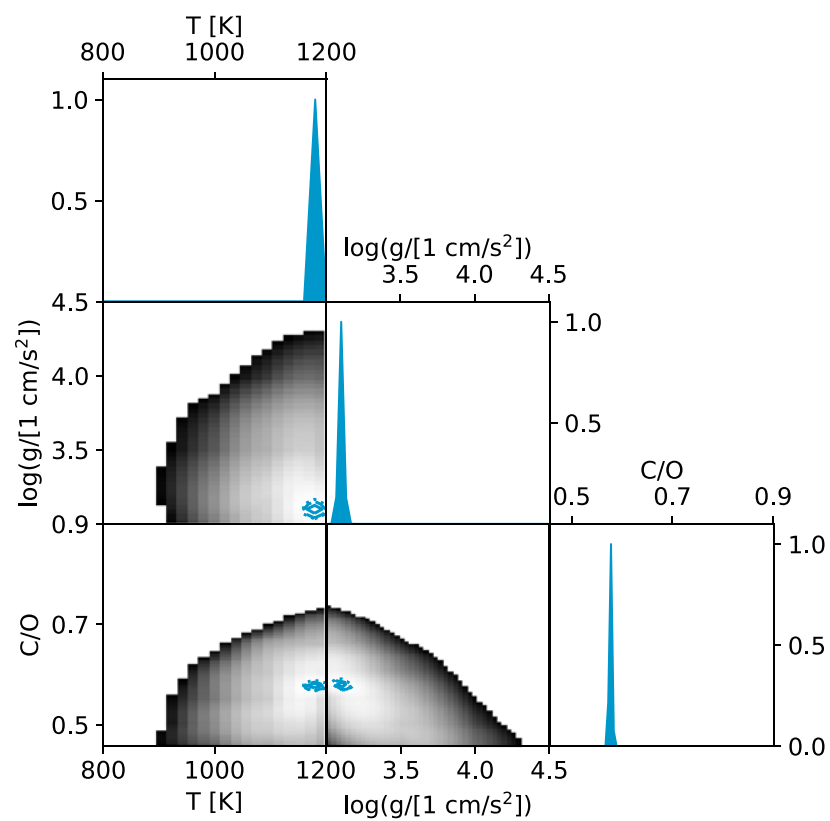

(a) HR 8799 b

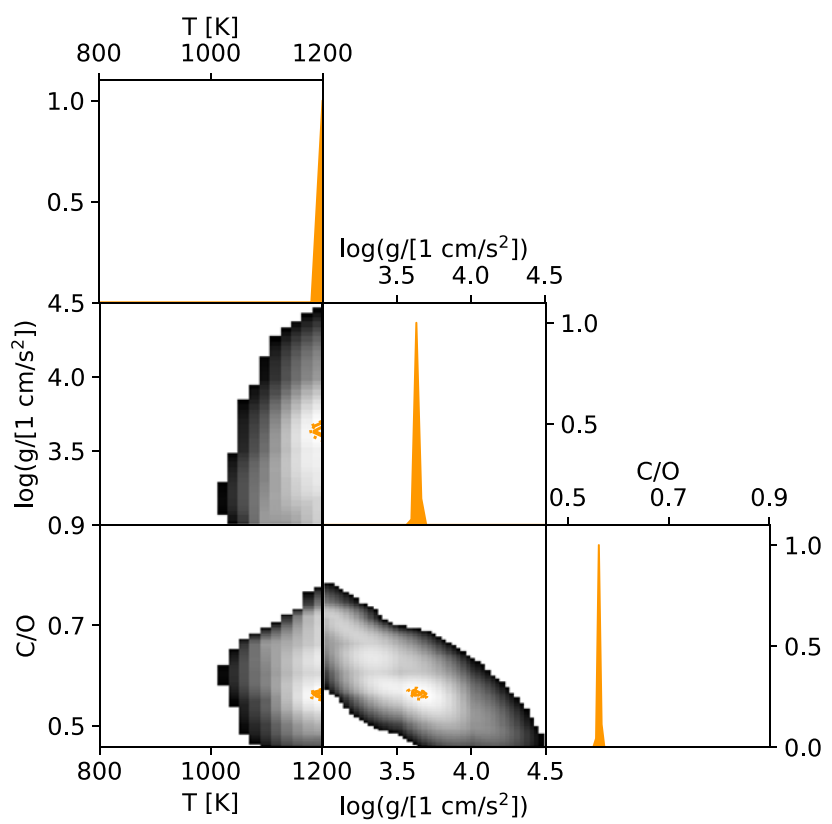

(b) HR 8799 c

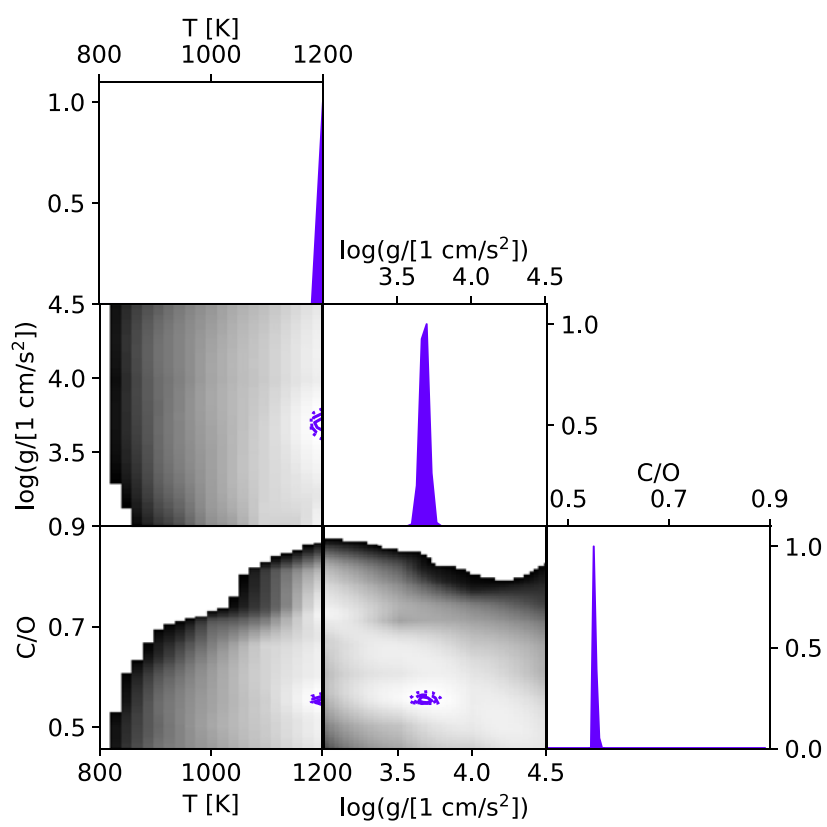

(2) HR 8799 d

Figure 19. Marginalized posteriors for $T_{\text {eff }}, \log (g)$, and C/O for $\mathrm{HR} 8799 \mathrm{~b}, \mathrm{c}$, and d. The $2 \mathrm{D}$ maps represent the logarithm of the posterior probability density and include the contours are the $68 \%, 68 \%$, and $99.7 \%$ contours. 


\section{Appendix D HR 8799 Methane Detection}

In light of the analysis of a subset of our OSIRIS data on HR 8799 b (18 of the 49 frames) by Petit dit de la Roche et al. (2018), we performed a minimal reanalysis of all of our data for this source following the prescription from Barman et al. (2011a), Konopacky et al. (2013), and Barman et al. (2015). The primary change is implementation of a simultaneous telluric extraction and absorption as was performed in Konopacky et al. (2013). We originally did not do this in Barman et al. (2015), because the speckles are significantly fainter at the location of HR 8799 b than HR 8799 c, and we were concerned about adding more noise to our spectrum. We also note that while the CCF was used to help us assess whether a molecule was detected in Barman et al. (2015), it was not used for any subsequent analysis. The calculation of methane abundance was based entirely on modeling the extracted spectra.

Our original, data pipeline-processed data cubes for $\mathrm{HR}$ 8799 b were first multiplied by the original telluric calibration spectrum used to remove the telluric features. Then, following Konopacky et al. (2013), we extracted a spectrum at every location in the data cube, avoiding only those pixels surrounding HR 8799 b. We median combined these spectra to obtain a spectrum of HR 8799A for each cube. We then interpolated over the $\operatorname{Br} \gamma$ absorption feature and divided the spectrum by a blackbody with the stellar temperature to get our telluric calibration spectrum. We then divided each cube by its extracted telluric spectrum.

The data cubes were then cleaned for speckles using an identical procedure to Barman et al. (2015), with our polynomial fitting procedure (note this type of analysis was not used elsewhere in this work). Upon removing the speckles, individual spectra were extracted from each data cube with a $3 \times 3$ spaxel box. All spectra were then median combined to obtain our final spectrum for analysis. To compute the CCF, we subtracted a smoothed version of the spectrum from our final spectrum to remove the continuum, as in Konopacky et al. (2013).

We then performed a cross-correlation of our continuumremoved spectrum with a pure methane template at the appropriate temperature and surface gravity. The results are shown in Figure 20, where the resulting detection of methane remains and is stronger than the original detection. To check whether residual tellurics were still playing a role in this detection, we generated 30 different "noise" spectra by randomly choosing different locations in each speckleprocessed cube to extract a spectrum from using a $3 \times 3$ spaxel box, avoiding the location of the planet. All randomly selected spectra were then combined to generate a final "noise" spectrum. We cross-correlated all 30 spectra with the same methane template, and found no peak in the CCF at zero like we see for HR $8799 \mathrm{~b}$. These functions are shown as thin gray lines in Figure 20.

Because our original CCF methodology returned a peak where our newer forward modeling approach did not return a statistically significant detection, we decided to verify the "detection" threshold in each method by using model spectra with known amounts of methane in them. We generated fake planets using these models and a Gaussian flux distribution with a FWHM and peak flux based on the real HR $8799 \mathrm{~b}$ in

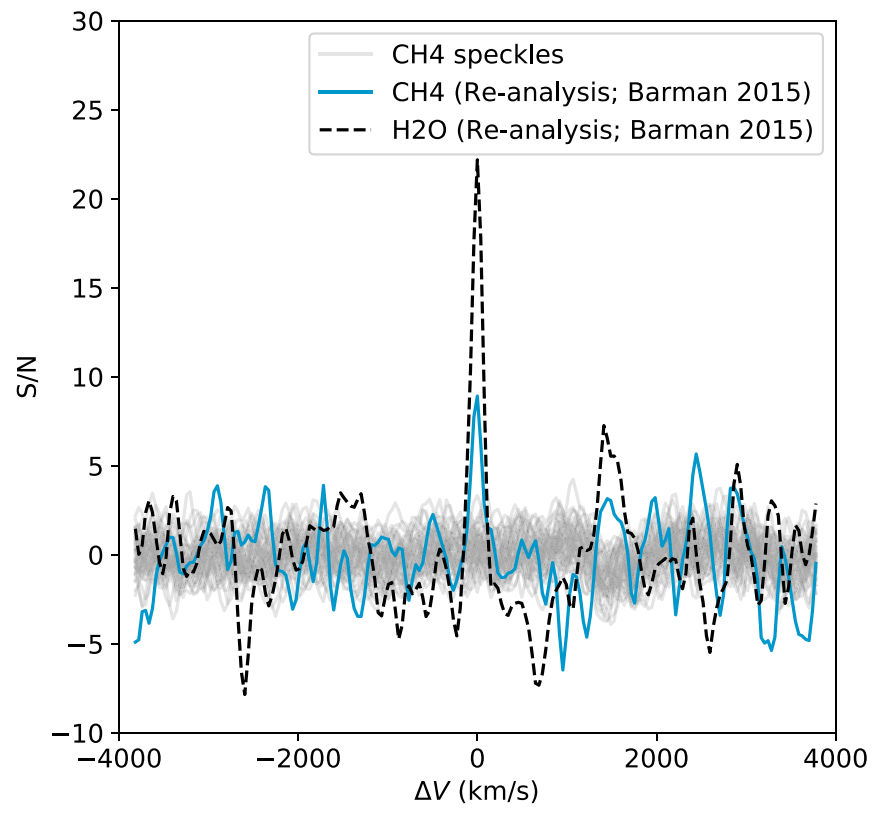

Figure 20. Revisited detection of $\mathrm{CH}_{4}$ and $\mathrm{H}_{2} \mathrm{O}$ in the atmosphere of $\mathrm{HR} 8799$ b with OSIRIS in $\mathrm{K}$ band from Barman et al. (2015). The $\mathrm{CH}_{4}$ cross correlation function (CCF) of the planet is compared to those of the speckles to estimate the $\mathrm{S} / \mathrm{N}$. We do not identify any systematics from the speckle field that could explain the methane signal of HR 8799 b.

the cubes. These were placed in each of our 49 reduced cubes at locations more than 7 spaxels away from the real planet. We tried this with three different models-one with a methane abundance based on our fits to the extracted spectra from Barman et al. (2015), one with twice that abundance of methane, and one with four times that abundance. We then ran the data cubes through each of our analysis pipelines, one following the method of Barman et al. (2015), and one following the methods presented in this paper. The peak of the methane $\mathrm{CCF}$ in both methods scaled with the relative abundance of methane, with the highest peaks for the $4 \mathrm{x}$ abundance of methane. When performing an analysis of the simulated data using the methodology presented in Section 4, we detected peaks for the two higher methane models with SNR equal to 8.8 and 12.8 . For the model that is similar to the HR 8799 b model from Barman et al. (2015), the peak in the center of the CCF has an SNR of 6.4, slightly higher than the value of 5.2 shown in Figure 1, which we do not claim as a detection. The difference in these two values is small, and suggests that a small amount of methane in the range of abundances given in Barman et al. (2015) is consistent with the methane CCF in this paper. The mole fraction of methane from fitting the extracted spectrum, including the continuum, was found to be $10^{-5.06}-10^{-5.85}$. Given that in our test we used a value in the middle of this range, it is certainly feasible that the abundance of methane is low enough in the spectrum to still be not give a statistically significant detection in our new method, as in Figure 1. Thus, we conclude that our result is consistent with the results of Barman et al. (2015) and does not preclude the presence of weak methane. Note that the lack of detection of methane in $H$ band was also the case in Barman et al. (2015) and was not inconsistent with our methane mole fraction, as the features are not expected to be stronger in $H$ than in $K$ band. 


\section{ORCID iDs}

Jean-Baptiste Ruffio (iD https://orcid.org/0000-00032233-4821

Quinn M. Konopacky (iD https://orcid.org/0000-00029936-6285

Travis Barman (iD https://orcid.org/0000-0002-7129-3002

Bruce Macintosh (ii) https://orcid.org/0000-0003-1212-7538

Kielan K. W. Hoch (iD https://orcid.org/0000-0002-9803-8255

Robert J. De Rosa (i) https://orcid.org/0000-0002-4918-0247

Jason J. Wang (i) https://orcid.org/0000-0003-0774-6502

Ian Czekala (i) https://orcid.org/0000-0002-1483-8811

Christian Marois (ib https://orcid.org/0000-0002-4164-4182

\section{References}

Allard, F., Homeier, D., \& Freytag, B. 2012, RSPTA, 370, 2765

Astropy Collaboration, Robitaille, T. P., Tollerud, E. J., et al. 2013, A\&A, 558, A33

Baines, E. K., White, R. J., Huber, D., et al. 2012, ApJ, 761, 57

Baranne, A., Queloz, D., Mayor, M., et al. 1996, A\&AS, 119, 373

Barman, T. S., Konopacky, Q. M., Macintosh, B., \& Marois, C. 2015, ApJ, 804,61

Barman, T. S., Macintosh, B., Konopacky, Q. M., \& Marois, C. 2011a, ApJ, 733, 65

Barman, T. S., Macintosh, B., Konopacky, Q. M., \& Marois, C. 2011b, ApJL, 735, L39

Bell, C. P. M., Mamajek, E. E., \& Naylor, T. 2015, MNRAS, 454, 593

Blunt, S., Ngo, H., Wang, J., et al. 2019, sblunt/orbitize: API Update to Prepare for RV+Astrometry Fits, Zenodo, doi:10.5281/zenodo.3337378

Blunt, S., Wang, J. J., Angelo, I., et al. 2020, AJ, 159, 89

Boehle, A., Larkin, J. E., Adkins, S. M., et al. 2016, Proc. SPIE, 9908, 99082Q

Bohlin, R. C., Gordon, K. D., \& Tremblay, P. E. 2014, PASP, 126, 711

Boley, A. C. 2009, ApJL, 695, L53

Bonnefoy, M., Zurlo, A., Baudino, J. L., et al. 2016, A\&A, 587, A58

Booth, M., Jordán, A., Casassus, S., et al. 2016, MNRAS, 460, L10

Booth, R. A., Clarke, C. J., Madhusudhan, N., \& Ilee, J. D. 2017, MNRAS, 469, 3994

Bosman, A. D., Cridland, A. J., \& Miguel, Y. 2019, A\&A, 632, L11

Boss, A. P. 1997, Sci, 276, 1836

Bowler, B. P., Blunt, S. C., \& Nielsen, E. L. 2020, AJ, 159, 63

Bowler, B. P., Liu, M. C., Dupuy, T. J., \& Cushing, M. C. 2010, ApJ, 723, 850

Brogi, M., de Kok, R. J., Birkby, J. L., Schwarz, H., \& Snellen, I. A. G. 2014, A\&A, 565, A124

Brogi, M., \& Line, M. R. 2019, AJ, 157, 114

Bryan, M. L., Benneke, B., Knutson, H. A., Batygin, K., \& Bowler, B. P. 2018, NatAs, 2, 138

Bryan, M. L., Chiang, E., Bowler, B. P., et al. 2020, AJ, 159, 181

Cale, B., Plavchan, P., LeBrun, D., et al. 2019, AJ, 158, 170

Contro, B., Horner, J., Wittenmyer, R. A., Marshall, J. P., \& Hinse, T. C. 2016, MNRAS, 463, 191

Cridland, A. J., Pudritz, R. E., Birnstiel, T., Cleeves, L. I., \& Bergin, E. A 2017, MNRAS, 469, 3910

Crossfield, I. J. M. 2014, A\&A, 566, A130

Crossfield, I. J. M., Biller, B., Schlieder, J. E., et al. 2014, Natur, 505, 654

Currie, T., Burrows, A., Girard, J. H., et al. 2014, ApJ, 795, 133

Currie, T., Burrows, A., Itoh, Y., et al. 2011, ApJ, 729, 128

Czekala, I., Andrews, S. M., Mandel, K. S., Hogg, D. W., \& Green, G. M. 2015, ApJ, 812, 128

Czekala, I., Mandel, K. S., Andrews, S. M., et al. 2017, ApJ, 840, 49

Fabrycky, D. C., \& Murray-Clay, R. A. 2010, ApJ, 710, 1408

Foreman-Mackey, D., Hogg, D. W., Lang, D., \& Goodman, J. 2013, PASP, 125,306

Gaia Collaboration, Brown, A. G. A., \& Vallenari, A. 2018, A\&A, 616, A1

Galicher, R., Marois, C., Macintosh, B., Barman, T., \& Konopacky, Q. 2011, ApJL, 739, L41

Gao, P., Plavchan, P., Gagné, J., et al. 2016, PASP, 128, 104501

Geiler, F., Krivov, A. V., Booth, M., \& Löhne, T. 2019, MNRAS, 483, 332

Gontcharov, G. A. 2006, AstL, 32, 759

Goździewski, K., \& Migaszewski, C. 2018, ApJS, 238, 6

Goździewski, K., \& Migaszewski, C. 2020, ApJL, 902, L40

Gravity Collaboration, Lacour, S., Nowak, M., et al. 2019, A\&A, 623, L11

Gravity Collaboration, Nowak, M., Lacour, S., et al. 2020, A\&A, 633, A110

Gray, R. O., \& Kaye, A. B. 1999, AJ, 118, 2993
Greenbaum, A. Z., Pueyo, L., Ruffio, J.-B., et al. 2018, AJ, 155, 226

Hinz, P. M., Rodigas, T. J., Kenworthy, M. A., et al. 2010, ApJ, 716, 417

Hoeijmakers, H. J., Ehrenreich, D., Heng, K., et al. 2018, Natur, 560, 453

Hughes, A. M., Wilner, D. J., Andrews, S. M., et al. 2011, ApJ, 740, 38

Hunter, J. D. 2007, CSE, 9, 90

Husser, T. O., Wende-von Berg, S., Dreizler, S., et al. 2013, A\&A, 553, A6

Ingraham, P., Marley, M. S., Saumon, D., et al. 2014, ApJL, 794, L15

Johansen, A., \& Lambrechts, M. 2017, AREPS, 45, 359

Karkoschka, E., \& Tomasko, M. G. 2010, Icar, 205, 674

Konopacky, Q. M., Barman, T. S., Macintosh, B. A., \& Marois, C. 2013, Sci, 339,1398

Konopacky, Q. M., Marois, C., Macintosh, B. A., et al. 2016, AJ, 152, 28

Krabbe, A., Gasaway, T., Song, I., et al. 2004, Proc. SPIE, 5492, 1403

Kratter, K. M., Murray-Clay, R. A., \& Youdin, A. N. 2010, ApJ, 710, 1375

Larkin, J., Barczys, M., Krabbe, A., et al. 2006, NewAR, 50, 362

Lavie, B., Mendonça, J. M., Mordasini, C., et al. 2017, AJ, 154, 91

Lee, J.-M., Heng, K., \& Irwin, P. G. J. 2013, ApJ, 778, 97

Lissauer, J. J. 1987, Icar, 69, 249

Lissauer, J. J., \& Kary, D. M. 1991, Icar, 94, 126

Lockhart, K. E., Do, T., Larkin, J. E., et al. 2019, AJ, 157, 75

Lyke, J., Do, T., Boehle, A., et al. 2017, OSIRIS Toolbox: OH-Suppressing InfraRed Imaging Spectrograph Pipeline, Astrophysics Source Code Library, ascl:1710.021

Madhusudhan, N., Bitsch, B., Johansen, A., \& Eriksson, L. 2017, MNRAS, 469, 4102

Madhusudhan, N., Burrows, A., \& Currie, T. 2011, ApJ, 737, 34

Malo, L., Doyon, R., Lafrenière, D., et al. 2013, ApJ, 762, 88

Marois, C., Correia, C., Galicher, R., et al. 2014, Proc. SPIE, 9148, 91480U

Marois, C., Macintosh, B., Barman, T., et al. 2008, Sci, 322, 1348

Marois, C., Zuckerman, B., Konopacky, Q. M., Macintosh, B., \& Barman, T. 2010, Natur, 468, 1080

Matthews, B., Kennedy, G., Sibthorpe, B., et al. 2014, ApJ, 780, 97

Mawet, D., Delorme, J. R., Jovanovic, N., et al. 2017, Proc. SPIE, 10400, 1040029

Mieda, E., Wright, S. A., Larkin, J. E., et al. 2014, PASP, 126, 250

Mollière, P., Stolker, T., Lacour, S., et al. 2020, A\&A, 640, A131

Mordasini, C., van Boekel, R., Mollière, P., Henning, T., \& Benneke, B. 2016, ApJ, 832, 41

Nielsen, E. L., De Rosa, R. J., Macintosh, B., et al. 2019, AJ, 158, 13

Öberg, K. I., Murray-Clay, R., \& Bergin, E. A. 2011, ApJL, 743, L16

Öberg, K. I., \& Wordsworth, R. 2019, AJ, 158, 194

O’Neil, K. K., Martinez, G. D., Hees, A., et al. 2019, AJ, 158, 4

Petit dit de la Roche, D. J. M., Hoeijmakers, H. J., \& Snellen, I. A. G. 2018, A\&A, 616, A146

Pueyo, L. 2016, ApJ, 824, 117

Pueyo, L., Soummer, R., Hoffmann, J., et al. 2015, ApJ, 803, 31

Qi, C., Öberg, K. I., Andrews, S. M., et al. 2015, ApJ, 813, 128

Read, M. J., Wyatt, M. C., Marino, S., \& Kennedy, G. M. 2018, MNRAS, 475, 4953

Reidemeister, M., Krivov, A. V., Schmidt, T. O. B., et al. 2009, A\&A, 503, 247

Ruffio, J.-B. 2019, PhD thesis, Stanford University, http://purl.stanford.edu/ yq071tj0740

Ruffio, J.-B., Macintosh, B., Konopacky, Q. M., et al. 2019, AJ, 158, 200

Schwarz, H., Ginski, C., de Kok, R. J., et al. 2016, A\&A, 593, A74

Skemer, A. J., Close, L. M., Szúcs, L., et al. 2011, ApJ, 732, 107

Skemer, A. J., Hinz, P. M., Esposito, S., et al. 2012, ApJ, 753, 14

Skemer, A. J., Marley, M. S., Hinz, P. M., et al. 2014, ApJ, 792, 17

Snellen, I. A. G., Brandl, B. R., de Kok, R. J., et al. 2014, Natur, 509, 63

Soummer, R., Hagan, J. B., Pueyo, L., et al. 2011, ApJ, 741, 55

Su, K. Y. L., Rieke, G. H., Stapelfeldt, K. R., et al. 2009, ApJ, 705, 314

Turrini, D., Schisano, E., Fonte, S., et al. 2021, ApJ, 909, 40

Vanderburg, A., Rappaport, S. A., \& Mayo, A. W. 2018, AJ, 156, 184

Vousden, W. D., Farr, W. M., \& Mandel, I. 2016, MNRAS, 455, 1919

Wang, J., Mawet, D., Fortney, J. J., et al. 2018a, AJ, 156, 272

Wang, J., Wang, J., Ma, B., et al. 2020, AJ, 160, 150

Wang, J. J., Graham, J. R., Dawson, R., et al. 2018b, AJ, 156, 192

Wang, J. J., Kulikauskas, M., \& Blunt, S. 2021, whereistheplanet: Predicting Positions of Directly Imaged Companions, Astrophysics Source Code Library, ascl:2101.003

Wertz, O., Absil, O., Gómez González, C. A., et al. 2017, A\&A, 598, A83 Wilcomb, K. K., Konopacky, Q. M., Barman, T. S., et al. 2020, AJ, 160, 207 Wilner, D. J., MacGregor, M. A., Andrews, S. M., et al. 2018, ApJ, 855, 56 Yurchenko, S. N., \& Tennyson, J. 2014, MNRAS, 440, 1649

Zhang, Z., Liu, M. C., Hermes, J. J., et al. 2020, ApJ, 891, 171

Zuckerman, B., Rhee, J. H., Song, I., \& Bessell, M. S. 2011, ApJ, 732, 61

Zurlo, A., Vigan, A., Galicher, R., et al. 2016, A\&A, 587, A57 